\title{
Symplectic Geometry of Entanglement
}

\author{
Adam Sawicki $^{1}$, Alan Huckleberry ${ }^{2}$, Marek Kuś ${ }^{1}$ \\ ${ }^{1}$ Center for Theoretical Physics, Polish Academy of Sciences, Al. Lotników 32/46, \\ 02-668 Warszawa, Poland. E-mail: assawi@cft.edu.pl; marek.kus@cft.edu.pl \\ ${ }^{2}$ Fakultät für Mathematik, Ruhr-Universität Bochum, 44780 Bochum, Germany
}

Received: 19 July 2010 / Accepted: 10 December 2010

Published online: 13 May 2011 - (C) The Author(s) 2011. This article is published with open access at Springerlink.com

\begin{abstract}
We present a description of entanglement in composite quantum systems in terms of symplectic geometry. We provide a symplectic characterization of sets of equally entangled states as orbits of group actions in the space of states. In particular, using the Kostant-Sternberg theorem, we show that separable states form a unique symplectic orbit, whereas orbits of entangled states are characterized by different degrees of degeneracy of the canonical symplectic form on the complex projective space. The degree of degeneracy may be thus used as a new geometric measure of entanglement. The above statements remain true for systems with an arbitrary number of components, moreover the presented method is general and can be applied also under different additional symmetry conditions stemming, e.g., from the indistinguishability of particles. We show how to calculate the degeneracy for various multiparticle systems providing also simple criteria of separability.
\end{abstract}

\section{Introduction}

Quantum entanglement - a direct consequence of linearity of quantum mechanics and the superposition principle - is one of the most intriguing phenomena distinguishing quantum and classical description of physical systems. Quantum states which are entangled possess features unknown in the classical world, like the seemingly paradoxical nonlocal properties exhibited by the famous Einstein-Podolsky-Rosen analysis of completeness of the quantum theory. Recently, with the development of quantum information theory they came to prominence as the main resource for several applications aiming at speeding up and making more secure information transfers (see, e.g., [1]).

Pure states which are not entangled are called separable and for systems of $L$ distinguishable particles they are, by definition, described by simple tensors in the Hilbert space of the whole system, $\mathcal{H}=\mathcal{H}_{1} \otimes \cdots \otimes \mathcal{H}_{L}$, where $\mathcal{H}_{l}$ are the single-particle spaces. For indistinguishable particles such a definition lacks sense - indistinguishability enforces symmetrization or antisymmetrization of the state vectors. In effect nearly all 
states are not simple tensors, in fact the relevant Hilbert spaces of such systems are no longer full tensor products, but rather their symmetric or antisymmetric subspaces. In these cases one modifies the original definition of separability and adapts it according to symmetry (see below).

The concept of separability (or equivalently nonentanglement) can be in a natural way extended to mixed states by first identifying pure states with projections on their directions (i.e. rank-one orthogonal projections) and then defining mixed separable states as convex combinations of pure separable ones. Mixed states which are not separable are, consequently, called entangled.

Separability of a state remains unaffected under a particular class of transformations allowed by quantum mechanics. Thus, for example, a separable state of distinguishable particles remains separable when we act on it by a unitary operator $U=U_{1} \otimes \cdots \otimes U_{L}$, where $U_{l}$ are unitaries acting in the single-particle spaces. One can find appropriate classes of unitary operators preserving separability also in the cases of indistinguishable particles. Going one step further one may analyze how actions of separability-preserving unitaries stratify into their orbits the whole space of states (pure or mixed) of a composite quantum system. To treat all the cases in a unified way we may consider a general situation in which a compact group $K$ acts on some manifold $M$. The manifold in question will then depend on the considered system. For pure states it will be the projectivisation $\mathbb{P}(\mathcal{H})$ of the Hilbert space $\mathcal{H}$ in the case of distinguishable particles or the projectivisation of an appropriate symmetrization (for bosons) or antisymmetrization (for fermions) of $\mathcal{H}$. In all cases the manifold $M$ is naturally equipped with some additional structure. In our investigations it will be a symplectic structure inherited from the natural one existing on every complex Hilbert space. Orbits of $K$ being submanifolds of $M$ might also, under special circumstances, inherit the symplectic structure or in addition respect the underlying complex structure of $\mathcal{H}$ and become Kählerian. From this point of view we want to consider several problems.

1. How symplectic and nonsymplectic orbits of the $K$ action on $\mathbb{P}(V)$ stratify the set of pure states?

2. What is the meaning (for the entanglement properties) of the fact that the orbit through a particular pure state is or is not symplectic?

In the next section we start with relevant definitions of separability and entanglement for distinguishable as well as indistinguishable particles. When giving definitions we concentrate on $L=2$, i.e. on two-partite systems, but the general reasonings for larger $L$ remains very similar. To make the paper reasonably self-contained we devote a few further sections and the Appendix to a presentation of some tools from the Lie-group representation theory and the symplectic geometry most important in our investigations.

\section{Separable and Entangled States}

Let $\mathcal{H}$ be an $N$-dimensional Hilbert space. By choosing an orthonormal basis in $\mathcal{H}$ we will identify it with $\mathbb{C}^{N}$ equipped with the standard Hermitian product.

A state is a positive, trace-one linear operator on $\mathcal{H}$,

$$
\rho: \mathcal{H} \rightarrow \mathcal{H}, \quad \forall_{x \in \mathcal{H}}\langle x|\rho| x\rangle \geq 0, \quad \operatorname{Tr} \rho=1 .
$$

We use the standard Dirac notation: $|x\rangle$ is an element of $\mathcal{H}$, and $\langle x|-$ the element of the dual space $\mathcal{H}^{*}$ corresponding to $|x\rangle$ via the scalar product $\langle\cdot \mid \cdot\rangle$ on $\mathcal{H}$. A state is, by 
definition, pure if it is a rank-one projection,

$$
\rho=\rho^{2}
$$

otherwise it is called mixed. A pure state can be thus written in the form $\rho=$ $|x\rangle\langle x| /\langle x \mid x\rangle:=P_{x}$ for some $x \in \mathcal{H}$, hence it can be identified with a point in the projective space $\mathbb{P}(\mathcal{H})$.

2.1. Separable and entangled states of two distinguishable particles. The Hilbert space for a composite system of two distinguishable particles is the tensor product of the Hilbert spaces of the subsytems,

$$
\mathcal{H}=\mathcal{H}_{1} \otimes \mathcal{H}_{2}, \quad \mathcal{H}_{1} \simeq \mathbb{C}^{N_{1}}, \quad \mathcal{H}_{2} \simeq \mathbb{C}^{N_{2}}
$$

A pure state $\rho$ is called separable or, equivalently, nonentangled if and only if it is a tensor product of pure states of the subsystems,

$$
\rho=P_{x} \otimes P_{y}, \quad|x\rangle \in \mathcal{H}_{1}, \quad|y\rangle \in \mathcal{H}_{2},
$$

otherwise it is called entangled. A mixed state is, by definition, separable if it is a convex combination of pure separable states [2],

$$
\rho=\sum_{i} p_{i} P_{x_{i}} \otimes P_{y_{i}}, \quad\left|x_{i}\right\rangle \in \mathcal{H}_{1}, \quad\left|y_{i}\right\rangle \in \mathcal{H}_{2}, \quad p_{i}>0, \quad \sum_{i} p_{i}=1 .
$$

From the physical point of view it is often desirable to define how strongly entangled is a particular state $\rho$. Although such a quantification of entanglement is not universal, especially for systems with more than two constituents and can be constructed on the basis of different (measured in an actual experiment) properties of entangled states, it should always ascribe the same amount of entanglement to states differeing by local quantum operations, i.e. by a conjugation by direct product of the unitary groups $U\left(\mathcal{H}_{1}\right) \times U\left(\mathcal{H}_{2}\right)$,

$$
\rho \mapsto U_{1} \otimes U_{2} \rho U_{1}^{\dagger} \otimes U_{2}^{\dagger}
$$

2.2. Separable and entangled states of two indistinguishable particles. For indistinguishable particles the Hilbert space of a composite two-partite system is no longer the tensor product of the Hilbert spaces of the subsystems but,

1. the antisymmetric part of the tensor product in the case of fermions,

$$
\mathcal{H}_{F}=\bigwedge^{2}\left(\mathcal{H}_{1}\right)
$$

2. the symmetric part of the tensor product in the case of bosons,

$$
\mathcal{H}_{B}=\operatorname{Sym}^{2}\left(\mathcal{H}_{1}\right)
$$


where $\mathcal{H}_{1} \simeq \mathbb{C}^{N}$ is the, so called, one-particle Hilbert space, i.e. the Hilbert space of a single particle.

In the fermionic state there is a natural way of defining pure nonentangled states: a state $\rho$ is nonentangled if and only if it is an orthogonal projection on an antisymmetric part of the tensor product of two vectors from $\mathcal{H}_{1}[3,4]$. Otherwise $\rho$ is called entangled. This definition, which can be in an obvious way extended to multipartite systems, is equivalent to the one proposed in [3] and [4].

Interestingly, a completely analogous definition for two bosons identifying nonentangled pure states with orthogonal projections on symmetrized tensor products of two vectors leads to some unexpected consequences - there are two classes of unentangled states inequivalent from the point of view of the action of the appropriate unitary group in the single particle spaces. Namely, to keep the indistinguishability intact, the unitary action of $U(N) \times U(N)$ on the Hilbert space of the composite system $\mathcal{H}_{B}$ given by Eq. (8) must be restricted to the diagonal one, i.e., involving the same unitary operator in both copies of the single particle space $\mathcal{H}_{1}$. Clearly, nonentangled states being products of two copies of the same state lie then on the other orbit of the unitary action than states which are (projections of) symmetrized tensor products of distinct (say, orthogonal) vectors from $\mathcal{H}_{1}$. As a consequence, two definitions supported by physical arguments were employed in the literature of the subject. In [5] (see also [6]) a concept of 'complete system of properties' of a subsystem was used to identify both above described classes of states as nonentangled, whereas in [7] it was pointed that only the restriction of the definition of nonentanglement to products of two copies of the same vector assures that nonentangled states can not be used to perform such a clearly 'nonclassical' task like e.g. teleportation, which definitely remains in accordance with the basic intuition connecting nonentanglement with the classical world. The second definition of nonentangled bosonic states was also proposed in [4], based on slightly different arguments. We will return to the problem in Sect. 10, provisionally adopting the more restrictive definition of nonentanglement in the bosonic case.

Mixed nonentangled states for fermions and bosons are defined, as in the case of distinguishable particles, as convex combinations of projections on pure nonentangled states.

As in the case of distinguishable particles the physically interesting amount of entanglement is invariant under the action of $U\left(\mathcal{H}_{1}\right)$ acting in the one-particle space $\mathcal{H}_{1}$.

\section{Pure Nonentangled States as Coherent States}

In all three cases of distinguishable particles, fermions, and bosons, the pure nonentangled states, treated as points in appropriate projective spaces, form a set invariant under the action of an appropriate compact, semisimple group $K$ irreducibly represented on some Hilbert space $\mathcal{H}$ [8-10]. This observation is in accordance with an intuition that entanglement properties of a state should not change under 'local' transformations allowed by quantum mechanics and symmetries of a system. Thus for example, for two distinguishable particles in two distant laboratories, local transformations can consist of independent quantum evolutions of each particle. This paradigm does not apply to indistinguishable particles when, in order to keep the exchange symmetry untouched, both particles must undergo the same evolution. Thus,

1. For distinguishable particles,

$$
K=S U\left(N_{1}\right) \times S U\left(N_{2}\right), \quad \mathcal{H}=\mathbb{C}^{N_{1}} \otimes \mathbb{C}^{N_{2}} .
$$


2. For fermions,

$$
K=S U(N), \quad \mathcal{H}=\bigwedge^{2}\left(\mathbb{C}^{N}\right)
$$

3. For bosons,

$$
K=S U(N), \quad \mathcal{H}=\operatorname{Sym}^{2}\left(\mathbb{C}^{N}\right)
$$

In all cases the nonentangled pure states are distinguished as forming some unique orbit of the underlying group action $[11,12]$. The orbit in question appears in the literature in several contexts and customarily its points are called coherent states, or the coherent states 'closest to classical states' [13]). A precise characteristic of the orbit, as well as its distinguished features from the view of entanglement theory will be discussed below.

\section{A Short Review of the Representation Theory}

Let us recall some fundamentals of the representation theory for semisimple Lie groups and algebras useful in the next sections [14].

In the following we denote by $K$ a simply connected compact Lie group and by $\mathfrak{k}$ its Lie algebra. It is standard fact that representations of $K$ are in one to one correspondence with representations of $\mathfrak{k}$. They both possess complete reducibility property, i.e., decompose as direct sums of irreducible ones, and can be made unitary by an appropriate choice of the scalar product in the carrier space. Let $\mathfrak{k}^{\mathbb{C}}$ be the complexification of $\mathfrak{k}$. It is also well known that irreducible representations of $\mathfrak{k}$ and $\mathfrak{k} \mathbb{C}$ are in one to one correspondence and that $\mathfrak{k}^{\mathbb{C}}$ is a semisimple complex Lie algebra.

Example 1. Consider $K=S U(N)$ which is simply connected and compact. Then $\mathfrak{k}=$ $\mathfrak{s u}_{N}$ and $\mathfrak{k}^{\mathbb{C}}=\mathfrak{s l}_{N}(\mathbb{C})$.

4.1. Adjoint representation of $\mathfrak{k}^{\mathbb{C}}$. The adjoint representation of $\mathfrak{k}^{\mathbb{C}}$ is defined as

$$
\begin{aligned}
& \operatorname{ad}: \mathfrak{k}^{\mathbb{C}} \rightarrow \mathfrak{g l}\left(\mathfrak{k}^{\mathbb{C}}\right), \\
& \operatorname{ad}_{X}(Y)=[X, Y] .
\end{aligned}
$$

This representation plays a key role in understanding all other representations of $\mathfrak{k}^{\mathbb{C}}$. Let us fix a maximal commutative subalgebra $\mathfrak{t}$ of $\mathfrak{k}$, then $\mathfrak{h}=\mathfrak{t}^{\mathbb{C}}=\mathfrak{t}+i \mathfrak{t}$ is a Cartan subalgebra of $\mathfrak{k}^{\mathbb{C}}$. Since $\mathfrak{h}$ is the maximal commutative subalgebra of $\mathfrak{k}^{\mathbb{C}}$ with the property that for every $H \in \mathfrak{h}$ the operator $\operatorname{ad}_{H}$ is diagonalizable (this is a consequence of the assumed semisimplicity of $K$ ), we can decompose $\mathfrak{k}^{\mathbb{C}}$ as a direct sum of root spaces with respect to $\mathfrak{h}$,

$$
\mathfrak{k}^{\mathbb{C}}=\mathfrak{h} \oplus \bigoplus_{\alpha} \mathfrak{g}_{\alpha}
$$

where $\alpha: \mathfrak{h} \rightarrow \mathbb{C}$ range over linear functionals (called roots) for which there exist $X \in \mathfrak{k}^{\mathbb{C}}$ such that

$$
\operatorname{ad}_{H}(X)=\alpha(H) X \quad \forall H \in \mathfrak{h} .
$$


Space $\mathfrak{g}_{\alpha}$ consists of the elements $X$ with the above property. It is a standard fact that if $\alpha$ is a root then $-\alpha$ is also a root and that $\left[\mathfrak{g}_{\alpha}, \mathfrak{g}_{\beta}\right]=0$ or $\left[\mathfrak{g}_{\alpha}, \mathfrak{g}_{\beta}\right]=\mathfrak{g}_{\alpha+\beta}$. Moreover all $\mathfrak{g}_{\alpha}$ are one dimensional. We may introduce the notion of a positive root by first choosing an arbitrary basis consisting of roots in the space spanned by them, and then defining positive roots as those with only positive coefficients in the decomposition in the chosen basis. The weight space decomposition of $\mathfrak{k}^{\mathbb{C}}$ can be then written as

$$
\mathfrak{k}^{\mathbb{C}}=\mathfrak{n}_{-} \oplus \mathfrak{h} \oplus \mathfrak{n}_{+},
$$

where the direct sums of the negative and positive root spaces, $\mathfrak{n}_{-}$and $\mathfrak{n}_{+}$are nilpotent Lie algebras. In the defining representation of $\mathfrak{s l}_{N}(\mathbb{C})$ as $N \times N$ complex traceless matrices, the most natural choice of positive roots is that which leads to $\mathfrak{n}_{-}$and $\mathfrak{n}_{+}$as, respectively, lower and upper triangular matrices.

It is a key fact that we can choose bases $E_{\alpha}$ of the root spaces $\mathfrak{g}_{\alpha}$ and define $H_{\alpha}=$ $\left[E_{-\alpha}, E_{\alpha}\right]$ so that $\left\{E_{-\alpha}, H_{\alpha}, E_{\alpha}\right\}$ is the standard basis for $\mathfrak{s l}_{2}(\mathbb{C})$. We will denote it by $\mathfrak{s l}_{2}(\alpha)$, by $\mathfrak{s u}_{2}(\alpha)$ the corresponding $\mathfrak{s u}_{2}$-triple $\left\{E_{-\alpha}-E_{\alpha}, i H_{\alpha}, i\left(E_{-\alpha}+E_{\alpha}\right)\right\}$, and by $S U_{2}(\alpha)$ the corresponding group.

4.2. General case. It is enough to restrict our attention to irreducible representations as $K$ is a compact and simply connected Lie group. Given any representation of $\mathfrak{h}$ on the complex vector space $V$ one decomposes $V$ as a direct sum:

$$
V=\oplus V_{\lambda}
$$

where isotypical components $V_{\lambda}$ are weight spaces. In other words:

$$
\xi . v=\lambda(\xi) v \quad \forall \xi \in \mathfrak{h} \text { and } v \in V_{\lambda},
$$

where the linear functionals $\lambda$ are called weights and vectors $v$ - the corresponding weight vectors. Every irreducible representation of $\mathfrak{k}^{\mathbb{C}}$ is the so-called highest weight cyclic representation. The most important facts we will use in the next sections are:

$-E_{\alpha} \cdot V_{\lambda} \subset V_{\lambda+\alpha}$

$-\left[E_{\alpha}, E_{\beta}\right] . V_{\lambda} \subset V_{\lambda+\alpha+\beta}$,

where $E_{\alpha} \in \mathfrak{g}_{\alpha}$ and $E_{\beta} \in \mathfrak{g}_{\beta}$.

\section{Symplectic Orbits of Group Actions}

In the following we will need a couple of facts about actions of Lie groups on symplectic manifolds (see e.g. [12]).

Let us denote by $(M, \omega)$ a symplectic manifold, i.e. $M$ is a manifold and $\omega$ is a nondegenerate, closed $(d \omega=0)$ two-form.

Let a compact semisimple group $K$ act on $M$ via syplectomorhisms, $K \times M \ni$ $(g, x) \mapsto \Phi_{g}(x) \in M, \Phi_{g}^{*} \omega=\omega$. We denote by $\mathfrak{k}^{*}$ the space dual to $\mathfrak{k}=\operatorname{Li} e(K)$.

Let $\xi \in \mathfrak{k}$. We define a vector field $\hat{\xi}$,

$$
\hat{\xi}(x)=\left.\frac{d}{d t}\right|_{t=0} \Phi_{\exp t \xi}(x) .
$$


Since the action of the group is Hamiltonian (which is true for a semisimple $K$ ), for each $\xi \in \mathfrak{k}$ there exists a Hamilton function $\mu_{\xi}: M \rightarrow \mathbb{R}$ for $\hat{\xi}$, i.e.

$$
d \mu_{\xi}=l_{\hat{\xi}} \omega:=\omega(\xi, \cdot)
$$

The function can be chosen to be linear in $\xi$, i.e.

$$
\mu_{\xi}(x)=\langle\mu(x), \xi\rangle, \quad \mu(x) \in \mathfrak{k}^{*},
$$

where $\langle$,$\rangle is the pairing between \mathfrak{k}$ and its dual $\mathfrak{k}^{*}$. The map $\mu_{\xi}$ defines thus by (21) a map $\mu: M \rightarrow \mathfrak{k}^{*}$. We can choose $\mu$ to be equivariant with respect to the coadjoint action of $K$ [15], i.e.

$$
\mu\left(\Phi_{g}(x)\right)=\operatorname{Ad}_{g}^{*} \mu(x),
$$

where the coadjoint action $\operatorname{Ad}_{g}^{*}$ on $\mathfrak{k}^{*}$ is defined via

$$
\left\langle\operatorname{Ad}_{g}^{*} \alpha, h\right\rangle=\left\langle\alpha, \operatorname{Ad}_{g^{-1}} h\right\rangle=\left\langle\alpha, g^{-1} h g\right\rangle, \quad g \in K, \quad h \in \mathfrak{k}, \quad \alpha \in \mathfrak{k}^{*},
$$

and Ad is the adjoint representation of $K$,

$$
\begin{gathered}
\operatorname{Ad}: K \rightarrow G l(\mathfrak{k}) \\
\operatorname{Ad}(g) X=g X g^{-1}:=\left.\frac{d}{d t}\right|_{t=0} g \exp t X g^{-1} .
\end{gathered}
$$

The above constructed $\mu$ is called the momentum map.

The goal is now to describe the criterion for the $K$-orbit to be symplectic. Let $\mathcal{O}=K . x$ be the orbit through a point $x \in M$. Denote by $\omega_{\mathcal{O}}$ the restriction of the symplectic form $\omega$ to $\mathcal{O}$. This form may, and in fact usually does, have a certain degree of degeneracy. Denote by $D_{x}$ the subspace of tangent vectors which are $\omega_{\mathcal{O}}$-orthogonal to the full space $T_{x} \mathcal{O}$. Since the $K$ action is symplectic we have $\Phi_{g *}\left(D_{x}\right)=D_{\Phi_{g}(x)}$ which means that the degree of degeneracy is constant on the orbit $\mathcal{O}$. This fact will turn out to be very important in the context of entanglement measures. Now because of $(22), \mu(\mathcal{O})=\Omega$ is a coadjoint orbit in $\mathfrak{k}^{*}$ and thus is symplectic with respect to the canonical form $\omega_{\Omega}$ on $\Omega$ (see the Appendix). We also have $\left(\left.\mu\right|_{\mathcal{O}}\right)^{*}\left(\omega_{\Omega}\right)=\omega_{\mathcal{O}}$, which means that the tangent spaces of the fibers of $\left.\mu\right|_{\mathcal{O}}$ are exactly the degeneracy spaces $D_{x}$. Indeed, if $u \in D_{x}$ then for an arbitrary $v \in T_{x} \mathcal{O}$,

$$
0=\omega_{\mathcal{O}}(u, v)=\omega_{\Omega}\left(\left(\left.\mu\right|_{\mathcal{O}}\right)_{*} u,\left(\left.\mu\right|_{\mathcal{O}}\right)_{*} v\right) .
$$

But $\omega_{\Omega}$ is nondegenerate and $T_{\mu(x)} \Omega=\left(\left.\mu\right|_{\mathcal{O}}\right)_{*} T_{x} \mathcal{O}$. Thus $\left(\left.\mu\right|_{\mathcal{O}}\right)_{*} u=0$ whenever $u \in D_{x}$. As a conclusion we get

Theorem 1. A $K$-orbit $\mathcal{O}=K . x$ in $M$ is symplectic if and only if the restriction of the moment map $\left.\mu\right|_{\mathcal{O}}$ is a diffeomorphism onto a coadjoint orbit $\Omega$.

Suppose now that $\mathcal{O}$ defined as above is symplectic. This means that $K$-action on $\mathcal{O}$ is the same as the coadjoint $K$-action on its $\mu$-image $\Omega$ (because $\mu$ is a diffeomorphism). Since $K$ is compact there exists an Ad-invariant scalar product $(\cdot \mid \cdot)$ on the carrier space $\mathfrak{k}$,

$$
(\operatorname{Ad}(g) X \mid \operatorname{Ad}(g) Y)=(X \mid Y), \quad \forall X, Y \in \mathfrak{k}, \quad g \in K
$$


In particular every operator $\operatorname{Ad}(g)$ is unitary, operators $\operatorname{ad}_{X}$ are anitHermitian $\left(\operatorname{ad}_{X}^{*}=\right.$ $\left.-\operatorname{ad}_{X}\right)$, and

$$
\left(\operatorname{ad}_{X} Y \mid Z\right)=-\left(Y \mid \operatorname{ad}_{X} Z\right) .
$$

We may use the invariant scalar product (26) to identify $\mathfrak{k}$ with $\mathfrak{k}^{*}$. More specifically we know that for any $\alpha \in \mathfrak{k}^{*}$ there exist $X \in \mathfrak{k}$ such that $\alpha=(X \mid \cdot)$. Upon such identification coadjoint orbits are exactly adjoint ones. To see this consider $\alpha \in \mathfrak{k}^{*}$. We know that

$$
\alpha=(X \mid \cdot) \equiv \alpha_{X}
$$

for some $X \in \mathfrak{k}$. We need to show that $\operatorname{Ad}_{g}^{*} \alpha$ (defined by 23) is equal to $\alpha_{\operatorname{Ad}(g) X}$. We have

$$
\begin{aligned}
\left\langle\operatorname{Ad}_{g}^{*} \alpha_{X}, Y\right\rangle & =\left\langle\alpha_{X}, \operatorname{Ad}\left(g^{-1}\right) Y\right\rangle=\left(X \mid \operatorname{Ad}\left(g^{-1}\right) Y\right) \\
& =(\operatorname{Ad}(g) X \mid Y)=\alpha_{\operatorname{Ad}(g) X}, \quad \forall g \in K,
\end{aligned}
$$

but this exactly what we wanted. Now we have the important fact which says that the adjoint action of $K$ on $\mathfrak{t}$ (the maximal commutative subalgebra of $\mathfrak{k}$ ) gives the whole $\mathfrak{k}$. This observation is true for any compact group but in the following we will need only its exemplification given by a familiar example.

Example 2. Let $K=S U(n)$ with the Lie algebra $\mathfrak{k}=\mathfrak{s u}$ of traceless antiHermitian matrices. Maximal commutative subalgebra of $\mathfrak{k}$ consists of traceless diagonal matrices $\mathfrak{t}=\operatorname{diag}\left(i t_{1}, \ldots, i t_{n}\right)$, where $t_{k} \in R$. It is a well known fact that every antiHermitian matrix has a purely imaginary spectrum and can be diagonalized by a unitary operator. Therefore, taking any $X \in \mathfrak{k}$ we can find $U \in U(n)$ such that

$$
U X U^{-1}=\operatorname{diag}\left(i t_{1}, \ldots, i t_{n}\right) \quad t_{k} \in \mathbb{R} \quad \forall k .
$$

Moreover we can choose $S U(n) \ni U_{1}=\operatorname{det}(U)^{-\frac{1}{n}} U$ so that $\operatorname{det}\left(U_{1}\right)=1$ and

$$
X=U_{1}^{-1} \operatorname{diag}\left(i t_{1}, \ldots, i t_{n}\right) U_{1} \quad t_{k} \in \mathbb{R} \quad \forall k .
$$

Hence indeed, every matrix $X \in \mathfrak{k}$ can be obtained from the $\mathfrak{t}$ by the adjoint action.

As a consequence we obtain that every adjoint orbit contains an element of the maximal commutative subalgebra $\mathfrak{t}$ which is fixed by the adjoint action of the maximal torus $T \subset G$, where torus $T$ is obtained by exponentiating $\mathfrak{t}\left(T=\left\{e^{t}: t \in \mathfrak{t}\right\}\right)$. Indeed, since $T$ is Abelian it fixes its elements by conjugation, $t t^{\prime} t^{-1}=t^{\prime}$, for $t, t^{\prime} \in T$. By differentiation it translates to fixing the elements of $t$ (and, consequently $\mathfrak{t}^{*}$ ) by the adjoint (coadjoint) action of $T$. Combining this observation with Theorem 1 establishing the diffeomorphism of a symplectic orbit with some coadjoint one, we arrive at the following conclusion

Theorem 2. If an orbit $\mathcal{O}$ of $K$ through $x \in M$ is symplectic then the set of points on $\mathcal{O}$ fixed by the action of $T$ is nonempty, $\operatorname{Fix}_{\mathcal{O}}(T) \neq 0$.

If the point $x \in M$ is fixed by an element $g \in K$ then by the equivariant property of the moment map, its $\mu$-image is also fixed by the adjoint action $\operatorname{Ad}(g)$. The degeneracy subspaces $D_{x}$ originate from nontrivial action of those symplectomorphisms $\Phi_{g}$ for which the corresponding $\operatorname{Ad}(g)$-action on $\mu(x)$ is trivial. Thus we have the following theorem $[11,12]$. 
Theorem 3. The orbit of $K$ through $x \in M$ is symplectic if and only if the stabilizer subgroup (of the K-action) of $x$ is the same as the stabilizer subgroup (of the $\mathrm{Ad}^{*}$-action) of $\mu(x)$.

It is always true that $\operatorname{Stab}(x) \subset \operatorname{Stab}(\mu(x))$, hence,

Corollary 1. The dimension of degeneracy subspace $D_{x}$ for an orbit $\mathcal{O}=K$.x does not depend on $x \in \mathcal{O}$ and can be computed as

$D(x)=\operatorname{dim}\left(D_{x}\right)=\operatorname{dim}(\operatorname{Stab}(x))-\operatorname{dim}(\operatorname{Stab}(\mu(x)))=\operatorname{dim}(K . x)-\operatorname{dim}(K . \mu(x))$.

This means we can associate with every orbit of $K$-action a non-negative integer $D(x)$ which measures the degree of its nonsymplecticity.

\section{Symplectic Orbits in the Space of States}

In the case of pure states $M=\mathbb{P}(V)$. The canonical symplectic form on $\mathbb{P}(V)$, the moment map and symplectic orbits of a unitary $K$ action can be calculated as follows $[11,12]$. For $A \in \mathfrak{u}(V)$ let $A_{x} \in T_{x} \mathbb{P}(V)$ be the vector tangent at $t=0$ to the curve $t \mapsto \pi(\exp (t A) v)$, where $x=\pi(v), v \in V,\|v\|=1$ and $\pi: V \rightarrow \mathbb{P}(V)$ is the canonical projection. When $A$ runs through the whole Lie algebra $\mathfrak{u}(V)$ the corresponding $A_{x}$ span $T_{x} \mathbb{P}(V)$ and for $A, B \in \mathfrak{u}(V)$ we obtain

$$
\omega_{x}\left(A_{x}, B_{x}\right)=-\operatorname{Im}\langle A v \mid B v\rangle=\frac{i}{2}\langle[A, B] v \mid v\rangle .
$$

The equivariant moment map $\mu: \mathbb{P}(V) \rightarrow \mathfrak{u}^{*}(V)$ for the action of $U(V)$ on $\mathbb{P}(V)$ is given by

$$
\mu_{A}(x)=\frac{1}{2}\langle v \mid A v\rangle .
$$

The group $K$ acts on $V$ via its unitary representation $\varrho: K \rightarrow U(V)$. The restriction of $\omega$ to $K . x$ can be calculated as above but now $A$ and $B$ are restricted to elements of $\mathfrak{k}$. From Sect. 5 we know that the necessary condition for an orbit to be symplectic is possessing a point fixed by the maximal torus $T$ of $K$. From the definition of weights and weight vectors (18) it easily follows that in the case of $K$-action via a unitary representation on a projective space $\mathbb{P}(V)$ fixed points of the $T$ action are exactly the weight vectors. Hence Theorem 2 can be reformulated as,

Fact 1. Let $K$ act on $\mathbb{P}(V)$ by unitary representation on a Hilbert space $V$. If $\mathcal{O}=K . x$ is a symplectic $K$-orbit then $\mathcal{O}$ contains a point $x=\pi(v)$, where $v$ is a $T$-weight vector, i.e., $v \in V_{\lambda}$ for some weight $\lambda$.

A sufficient condition for $\mathcal{O}=K . x$ to be symplectic is, of course, given in Theorem 3 but we want to have it in a more useful form. It is enough to restrict our attention to orbits passing through the weight vectors. For $v \in V_{\lambda}$ we consider the tangent space $T_{x} \mathcal{O}$ equipped with the 2 -form $\omega_{x}$. Let $\alpha$ be a positive root and define $\mathcal{O}_{\alpha}$ to be the orbit of $S U_{2}(\alpha)$ of the associated $S U_{2}$-triple. Let $P_{\alpha}$ denote the tangent space to $\mathcal{O}_{\alpha}$ at the point $x$. The tangent space $T_{x} \mathcal{O}$ can be of course considered as the collection of $P_{\alpha}$ where $\alpha$ 
range over all positive roots. Let $\mathfrak{k}^{\mathbb{C}}$ be the complexification of $\mathfrak{k}$ - the Lie algebra of $K$. It has the root-space decomposition

$$
\mathfrak{k}^{\mathbb{C}}=\mathfrak{t}^{\mathbb{C}} \underset{\alpha}{\oplus} \mathbb{C} E_{\alpha},
$$

where $E_{\alpha}$ is a root vector corresponding to the root $\alpha$, hence $\left[E_{\alpha}, E_{-\alpha}\right]=H_{\alpha} \in \mathfrak{t}^{\mathbb{C}}$. The corresponding decomposition of $\mathfrak{k}$ reads

$$
\mathfrak{k}=\mathfrak{t} \bigoplus_{\alpha} \mathbb{R}\left(E_{\alpha}-E_{-\alpha}\right) \underset{\alpha}{\oplus} \mathbb{R} i\left(E_{\alpha}+E_{-\alpha}\right),
$$

where $\alpha$ ranges over all positive roots.

Fact 2. If $\alpha$ and $\beta$ are different positive roots, then the tangent planes $P_{\alpha}$ and $P_{\beta}$ are $\omega$-orthogonal.

Proof. The symplectic form $\omega_{x}$ is given as:

$$
\omega_{x}\left(A_{x}, B_{x}\right)=\frac{i}{2}\langle[A, B] v \mid v\rangle .
$$

We have assumed that $v \in V_{\lambda}$. If $[A, B] v$ is in some other weight space, the right-hand side of (37) vanishes since two different weight spaces are orthogonal. We know that $P_{\alpha}=\operatorname{Span}\left\{\left(E_{\alpha}-E_{-\alpha}\right) \cdot v, i\left(E_{\alpha}+E_{-\alpha}\right) \cdot v\right\}$ and $P_{\beta}=\operatorname{Span}\left\{\left(E_{\beta}-E_{-\beta}\right) \cdot v, i\left(E_{\beta}+\right.\right.$ $\left.\left.E_{-\beta}\right) . v\right\}$. We also know that $\left[E_{\alpha}, E_{\beta}\right] . v \in V_{\lambda+\alpha+\beta}$ or is equal to zero. But $V_{\lambda+\alpha+\beta}$ is orthogonal to $V_{\lambda}$. Consequently, if $A_{x} \in P_{\alpha}$ and $B_{x} \in P_{\beta}$, then

$$
\omega_{x}\left(A_{x}, B_{x}\right)=\frac{i}{2}\langle[A, B] v \mid v\rangle=0,
$$

which is what we wanted to prove.

Summing up we know that $T_{x} \mathcal{O}=\bigcup_{\alpha} P_{\alpha}$ and that the spaces $P_{\alpha}$ are $\omega$-orthogonal. Hence $T_{x} \mathcal{O}$ is a symplectic vector space if and only if all $P_{\alpha}$ are symplectic.

Fact 3. The space $P_{\alpha}$ is symplectic if and only if $\left\langle\left[E_{\alpha}, E_{-\alpha}\right] v \mid v\right\rangle \neq 0$.

Proof. Let $A_{x} \in P_{\alpha}$ and $B_{x} \in P_{\alpha}$. Computing

$$
\omega_{x}\left(A_{x}, B_{x}\right)=\frac{i}{2}\langle[A, B] v \mid v\rangle,
$$

we see that only the term $\left\langle\left[E_{\alpha}, E_{-\alpha}\right] v \mid v\right\rangle$ can give a nonzero result and when it indeed does not vanish then $P_{\alpha}$ is symplectic which is what we wanted to prove.

Thus $T_{x} \mathcal{O}$ is symplectic when the following implication is true

$$
\left\langle\left[E_{\alpha}, E_{-\alpha}\right] v \mid v\right\rangle=0 \Rightarrow P_{\alpha}=0 .
$$

The left-hand side of (40) can be rewritten as

$$
\left[E_{\alpha}, E_{-\alpha}\right] v=H_{\alpha} \cdot v=\lambda\left(H_{\alpha}\right) v,
$$

where $\lambda$ is the weight of $v$. For the right-hand side of (40) recall that $P_{\alpha}=\operatorname{Span}\left\{\left(E_{\alpha}-\right.\right.$ $\left.\left.E_{-\alpha}\right) . v, i\left(E_{\alpha}+E_{-\alpha}\right) . v\right\}$. So $P_{\alpha}=0$ means $E_{\alpha} v=0=E_{-\alpha} v$. Hence [11], 
Theorem 4 (Kostant-Sternberg). The orbit $\mathcal{O}=K . x, x=\pi(v), v \in V_{\lambda}$ for some weight $\lambda$, is symplectic if and only if for every positive root $\alpha$ with $\lambda\left(H_{\alpha}\right)=0$ it follows that $E_{\alpha} v=0=E_{-\alpha} v$.

To demonstrate how this theorem works we will prove that the orbit through the highest weight vector is always symplectic. As it was mentioned in Subsect. 4.2 every unitary irreducible representation of the compact semisimple group $K$ is a highest weight representation. The highest weight vector is defined as follows

Definition 1. Let $K$ be compact semisimple Lie group and denote by $\mathfrak{k}$ its Lie algebra and by $\mathfrak{k}^{\mathbb{C}}$ its complexification. Then $\mathfrak{k}^{\mathbb{C}}$ admits decomposition (35). The weight vector $v \in V_{\lambda}$ of irreducible representation of $K$ (respectively $\mathfrak{k}$ or $\mathfrak{k}^{\mathbb{C}}$ ) is highest weight if and only if

$$
E_{\alpha} \cdot v=0
$$

where $\alpha$ range through all positive roots.

Let us take $v \in V_{\lambda}$ - the highest weight vector of irreducible representation of $\mathfrak{k}^{\mathbb{C}}$ and consider the corresponding orbit $\mathcal{O}=K . x$, where $x=\pi(v)$. It is easy to see that according to Definition $1, v$ is also the highest weight vector for all $\mathfrak{s l}_{2}(\alpha)$-triples. From the representation theory we know that weights of an irreducible representation of $\mathfrak{s l}_{2}(\alpha)$ are $W=\{-n,-n+2, \ldots, n-2, n\}$, where $n \geq 0$. This means

$$
H_{\alpha} \cdot v=\lambda\left(H_{\alpha}\right) v=n v .
$$

So $\lambda\left(H_{\alpha}\right)=n$. The only interesting $\alpha$ is the one for which $n=0$. But then we have a one dimensional, hence trivial, representation of $\mathfrak{s l}_{2}(\alpha)$. This, of course, means $E_{-\alpha} \cdot v=0=E_{\alpha} \cdot v$. Making use of Theorem 4 we see that $\mathcal{O}$ is symplectic. In fact this orbit is not only symplectic but also Kähler (see the Appendix). Indeed, from Theorem 6 we know that to prove this, it is enough to check that this orbit is complex manifold since $\mathbb{P}(V)$ is a positive Kähler manifold (see the Appendix). But if $v$ is the highest weight vector then the tangent space $T_{x} \mathcal{O}=\bigcup_{\alpha} P_{\alpha}$, where $\alpha$ range over positive roots and $P_{\alpha}=\operatorname{Span}\left\{E_{-\alpha} \cdot v, i E_{-\alpha} \cdot v\right\}$. So $T_{x} N$ is stable under multiplication by $i$, hence $\mathcal{O}$ is complex.

\section{Distinguishable Particles}

7.1. Two qubits case. In the simplest case of two qubits we may use directly the Kostant-Sternberg theorem from the last section. The Hilbert space is then $\mathcal{H}=\mathbb{C}^{2} \otimes \mathbb{C}^{2}$ and the direct product $K=S U(2) \times S U(2)$ acts on $\mathcal{H}$ in a natural way,

$$
\left(g_{1}, g_{2}\right) v_{1} \otimes v_{2}=g_{1} v_{1} \otimes g_{2} v_{2},
$$

where $g_{1}, g_{2} \in S U(2)$ and $v_{1}, v_{2} \in \mathbb{C}^{2}$. Our first goal is to identify symplectic orbits of $K$. To apply theorems and facts established in the previous sections we start with the root-space decomposition of the Lie algebra $\mathfrak{k}^{\mathbb{C}}=\mathfrak{s l}_{2}(\mathbb{C}) \oplus \mathfrak{s l}_{2}(\mathbb{C})$, i.e., the complexification of $\mathfrak{k}=\mathfrak{s u}_{2} \oplus \mathfrak{s u}_{2}$ - the Lie algebra of $G$. The algebra $\mathfrak{k}^{\mathbb{C}}$ is semisimple as a direct sum of the simple algebras $\mathfrak{s l}_{2}(\mathbb{C})$. Let us recall that

$$
\begin{aligned}
& \mathfrak{s l}_{2}(\mathbb{C})=\operatorname{Span}\{X, H, Y\}, \\
& {[H, X]=2 X, \quad[H, Y]=-2 Y, \quad[X, Y]=H .}
\end{aligned}
$$


The Cartan subalgebra of $\mathfrak{s l}_{2}(\mathbb{C})$ is spanned by $H$, whereas $\operatorname{Span}\{X\}, \operatorname{Span}\{Y\}$ are the positive and negative root spaces. An element of $\mathfrak{k}^{\mathbb{C}}$ can be written as $\left(Z_{1}, Z_{2}\right)$, where $Z_{1}, Z_{2} \in \mathfrak{s l}_{2}(\mathbb{C})$. We also have:

$$
\left[\left(Z_{1}, Z_{2}\right),\left(W_{1}, W_{2}\right)\right]=\left(\left[Z_{1}, W_{1}\right],\left[Z_{2}, W_{2}\right]\right)
$$

Knowing this we find that the Cartan subalgebra of $\mathfrak{k}^{\mathbb{C}}$ is $\mathfrak{t}=\operatorname{Span}\{(H, 0),(0, H)\}$. The commutation relations read as,

$$
\begin{aligned}
& {[(H, 0),(X, 0)]=2(X, 0), \quad[(H, 0),(Y, 0)]=-2(Y, 0), \quad[(X, 0),(Y, 0)]=(H, 0),} \\
& {[(0, H),(0, X)]=2(0, X), \quad[(0, H),(0, Y)]=-2(0, Y), \quad[(0, X),(0, Y)]=(0, H),} \\
& {[(0, W),(Z, 0)]=0 .}
\end{aligned}
$$

Since $\mathfrak{k}^{\mathbb{C}}$ is semisimple, its root spaces are one-dimensional. We have the following roots (computed in the basis $\{(H, 0),(0, H)\}$ of the Cartan subalgebra $\mathfrak{t}$ ), and the corresponding root spaces,

$$
\begin{aligned}
\alpha & =(2,0), \quad V_{\alpha} & =\operatorname{Span}\{(X, 0)\}, \\
-\alpha & =(-2,0), \quad V_{-\alpha} & =\operatorname{Span}\{(Y, 0)\}, \\
\beta & =(0,2), \quad V_{\beta} & =\operatorname{Span}\{(0, X)\}, \\
-\beta & =(0,-2), \quad V_{-\beta} & =\operatorname{Span}\{(0, Y)\} .
\end{aligned}
$$

Thus we have the following decomposition of $\mathfrak{k}^{\mathbb{C}}$,

$$
\begin{aligned}
\mathfrak{k}^{\mathbb{C}} & =\mathfrak{n}_{-} \oplus \mathfrak{t} \oplus \mathfrak{n}_{+}, \\
\mathfrak{n}_{-} & =\operatorname{Span}\{(Y, 0),(0, Y)\}, \\
\mathfrak{n}_{+} & =\operatorname{Span}\{(X, 0),(0, X)\}, \\
\mathfrak{t} & =\operatorname{Span}\{(H, 0),(0, H)\},
\end{aligned}
$$

where $\mathfrak{n}_{-}$and $\mathfrak{n}_{+}$are negative and positive root spaces, respectively. Let

$$
e_{1}=\left(\begin{array}{l}
1 \\
0
\end{array}\right), \quad e_{2}=\left(\begin{array}{l}
0 \\
1
\end{array}\right)
$$

be the standard basis of $\mathbb{C}^{2}$. The Lie algebra $\mathfrak{s l}_{2}(\mathbb{C})$ acts then via the defining representation,

$$
H=\left(\begin{array}{cc}
1 & 0 \\
0 & -1
\end{array}\right), \quad X=\left(\begin{array}{ll}
0 & 1 \\
0 & 0
\end{array}\right), \quad Y=\left(\begin{array}{ll}
0 & 0 \\
1 & 0
\end{array}\right) .
$$

The highest weight vector equals $e_{1}$ and there are just two weight spaces, one spanned by $e_{1}$ and the other by $e_{2}$. The corresponding weights are 1 and -1 .

The action of $\left(Z_{1}, Z_{2}\right) \in \mathfrak{k}^{\mathbb{C}}$ on $\mathcal{H}$ is given by

$$
\left(Z_{1}, Z_{2}\right) v_{1} \otimes v_{2}=Z_{1} v_{1} \otimes v_{2}+v_{1} \otimes Z_{2} v_{2} .
$$

It is easy to guess that the highest weight vector for the above representation equals $e_{1} \otimes e_{1}$. Indeed, it is an eigenvector of the Cartan subalgebra and is annihilated by all 
elements of $\mathfrak{n}_{+}$. The weight spaces are obtained by successive action of $\mathfrak{n}_{-}$on $e_{1} \otimes e_{1}$. In the basis $\{(H, 0),(0, H)\}$ the weights and weight vectors read as,

$$
\begin{array}{ll}
\lambda_{1}=(1,1), & v_{1}=e_{1} \otimes e_{1}, \\
\lambda_{2}=(1,-1), & v_{2}=e_{1} \otimes e_{2}, \\
\lambda_{3}=(-1,1), & v_{3}=e_{2} \otimes e_{1}, \\
\lambda_{4}=(-1,-1), & v_{4}=e_{2} \otimes e_{2} .
\end{array}
$$

The $\mathfrak{s l}_{2}(\mathbb{C})$ triples corresponding to the positive roots of $\mathfrak{k}^{\mathbb{C}}$ are $\{(X, 0),(H, 0),(Y, 0)\}$ and $\{(0, X),(0, H),(0, Y)\}$. To decide if an orbit through a weight vector is symplectic it is enough to check if $\lambda((0, H)) \neq 0$ and $\lambda((H, 0))) \neq 0$, where $\lambda$ is one of the weights from the list above. Since weights are given by two nonzero numbers $\left(n_{1}, n_{2}\right)$, we find

Fact 4. In the case of two qubits, only the orbits through weight vectors are symplectic in the projective space $\mathbb{P}\left(\mathbb{C}^{2} \otimes \mathbb{C}^{2}\right)$. In fact all weight vectors lie on the same orbit which is Kähler and contains all separable states. Orbits through entangled states are not symplectic

Let us now consider the states $e_{1} \otimes e_{2} \pm e_{2} \otimes e_{1}$ which are not weight vectors and are not separable. The orbits through them are not symplectic and we can ask what is the dimension of the degeneracy subspace for them. We need to examine which vectors from the tangent space to the orbit of $S U(2) \times S U(2)$ are tangent to the fibers of the corresponding moment map $\mu$. We already know that

$$
\mu_{A}(x)=\frac{1}{2}\langle v \mid A v\rangle, \quad x=\pi(v) .
$$

In our case we have,

$$
\begin{aligned}
\mu_{\left(Z_{1}, Z_{2}\right)}(x) & =\frac{1}{2}\left\langle e_{1} \otimes e_{2} \pm e_{2} \otimes e_{1} \mid\left(Z_{1}, Z_{2}\right)\left(e_{1} \otimes e_{2} \pm e_{2} \otimes e_{1}\right)\right\rangle \\
& =\operatorname{tr}\left(Z_{1}\right)+\operatorname{tr}\left(Z_{2}\right)=0, \quad\left(Z_{1}, Z_{2}\right) \in \mathfrak{k},
\end{aligned}
$$

where the first equality was obtained by a direct computation and the second one is a consequence of the zero-trace property of the matrices from $\mathfrak{s u}_{2}$. Thus the degeneracy space is the whole tangent space to the orbit through state $e_{1} \otimes e_{2} \pm e_{2} \otimes e_{1}$. This space can be directly computed, as it is spanned by the projection of vectors given by

$$
\left(Z_{1}, Z_{2}\right)\left(e_{1} \otimes e_{2} \pm e_{2} \otimes e_{1}\right)=Z_{1} e_{1} \otimes e_{2}+e_{1} \otimes Z_{2} e_{2} \pm Z_{1} e_{2} \otimes e_{1} \pm e_{2} \otimes Z_{2} e_{2} .
$$

Using the Pauli matrices multiplied by the imaginary unit $i$ as a basis for $\mathfrak{s u}_{2}$ and the formula (57) we obtain that in both cases the tangent space is three dimensional. In the case of the state $e_{1} \otimes e_{2}+e_{2} \otimes e_{1}$ it is spanned by $\left\{i\left(e_{1} \otimes e_{1}+e_{2} \otimes e_{2}\right),\left(e_{2} \otimes e_{2}-\right.\right.$ $\left.\left.e_{1} \otimes e_{1}\right), i\left(e_{1} \otimes e_{2}-e_{2} \otimes e_{1}\right)\right\}$, whereas for the state $e_{1} \otimes e_{2}-e_{2} \otimes e_{1}$ it is spanned by $\left\{i\left(e_{2} \otimes e_{2}-e_{1} \otimes e_{1}\right),\left(e_{1} \otimes e_{1}+e_{2} \otimes e_{2}\right), i\left(e_{1} \otimes e_{2}+e_{2} \otimes e_{1}\right)\right\}$. The conclusion is that we can use the dimension of the degeneracy space as a measure of entanglement.

In principle a similar reasoning directly using the Kostant-Sternberg theorem can be applied in cases of larger dimensions of subsystems and/or for many-partite systems involving multiple tensor products of spaces with arbitrary dimensions, but explicit calculations become prohibitively complicated. In the next section we present a method allowing for finding the degeneracy spaces for bipartite systems of arbitrary dimensions based on the Singular Value Decomposition (SVD) of a matrix, and in the following one we show how to extend the reasoning to a multipartite case where the direct application of SVD is not possible. 


\section{Degeneracy Subspaces and SVD}

The method of determining the dimension of the degeneracy space presented in the previous section can be extended to a more general case of two distinguishable particles, but in this case one can achieve the goal in a less cumbersome manner by invoking the Singular Value Decomposition of an arbitrary complex matrix [16]. We will present the solution for two distinguishable but otherwise identical particles (i.e., living in spaces of the same dimension $N$ ). A generalization to unequal dimensions of the spaces needs only a little bit more effort.

The Hilbert space is thus now $\mathcal{H}=\mathbb{C}^{N} \otimes \mathbb{C}^{N}$. Let us fix an orthonormal basis $\left\{e_{i}: i=1, \ldots, N\right\}$ of $\mathbb{C}^{N}$, (e.g., the standard one where $e_{i}$ is a column vector with one on the $i^{\text {th }}$ position and zero on others). Any state $|\Psi\rangle \in \mathcal{H}$ can be decomposed as:

$$
|\Psi\rangle=\sum_{i, j=1}^{N} C_{i j} e_{i} \otimes e_{j} .
$$

The action of $U \otimes V \in S U(N) \times S U(N)$ gives:

$$
\begin{aligned}
U \otimes V|\Psi\rangle=\sum_{i, j=1}^{N} C_{i j} U e_{i} \otimes V e_{j} & =\sum_{i, j=1}^{N} C_{i j} U_{k i} e_{k} \otimes V_{l j} e_{l} \\
& =\sum_{k, l=1}^{N}\left(U C V^{T}\right)_{k l} e_{k} \otimes e_{l} .
\end{aligned}
$$

It is a well known fact that any complex matrix can be put to a diagonal form by the simultaneous left and right action of the unitary group achieving the SVD, i.e. there exist unitary $\tilde{U}, \tilde{V}$ such that

$$
\tilde{U} C \tilde{V}=\operatorname{diag}\left(0, \ldots, 0, v_{1}, \ldots, v_{2}, \ldots, v_{K}\right),
$$

where $v_{i}>0$ and $\left\{0, \ldots, 0, v_{1}^{2}, \ldots, v_{2}^{2}, \ldots, v_{K}^{2}\right\}$ constitute the spectrum of $C^{\dagger} C$ (and, equivalently, the spectrum of $C C^{\dagger}$ ). Taking $U=\tilde{U}$ and $V=\tilde{V}^{T}$ in (67) we conclude that the orbit of $S U(N) \times S U(N)$ through any state $|\Psi\rangle$ contains a point which can be written as:

$$
\left|\Psi^{\prime}\right\rangle=\sum_{i=1}^{N} p_{i} e_{i} \otimes e_{i}
$$

with $p_{i} \geq 0$ and $\sum_{i=1}^{N} p_{i}^{2}=1$.

We denote by $m_{i}$ the multiplicity of $v_{i}$ and by $m_{0}$ the dimension of the kernel of $C$, hence $m_{0}+\sum_{n=1}^{K} m_{i}=N$. We can use the state $\left|\Psi^{\prime}\right\rangle$ to compute the dimension of the orbit through $|\Psi\rangle$. Crucial for this is the observation [17] that $\left|\Psi^{\prime}\right\rangle$ is stabilized by the action of $U \otimes V$ where:

$$
U=\left(\begin{array}{cccc}
u_{0} & & & \\
& u_{1} & & \\
& & \ddots & \\
& & & u_{K}
\end{array}\right), \quad V=e^{i \phi}\left(\begin{array}{cccc}
v_{0} & & & \\
& \bar{u}_{1} & & \\
& & \ddots & \\
& & & \bar{u}_{K}
\end{array}\right) \text {, }
$$


$u_{1}, \ldots, u_{K}$ are arbitrary unitary operators from, respectively, $U\left(m_{1}\right), \ldots, U\left(m_{K}\right)$. Both $u_{0}$ and $v_{0}$ belong to $U\left(m_{0}\right)$ and $\operatorname{det}\left(u_{0}\right)$ and $\operatorname{det}\left(v_{0}\right)$ are fixed by the determinants of $u_{1}, \ldots, u_{K}$ in a way ensuring that matrices $U, V$ are special unitary. Knowing this we can compute the dimension of the orbit $\mathcal{O}$ of $G=S U(N) \times S U(N)$ through $|\Psi\rangle$ in the projective space $\mathbb{P}(\mathcal{H})$ as:

$$
\operatorname{dim}(\mathcal{O})=\left(2 N^{2}-2\right)-\left(\left(2 m_{0}^{2}-2\right)+1+\sum_{n=1}^{K} m_{n}^{2}\right)=2 N^{2}-2 m_{0}^{2}-\sum_{n=1}^{K} m_{n}^{2}-1
$$

where we used $\operatorname{dim}(U(n))=n^{2}=\operatorname{dim}(S U(n))+1$. The dimensions of the two $U\left(m_{0}\right)$ blocks are diminished by one due to the determinant fixing condition stated above, and an additional one is subtracted due to the projection on $\mathbb{P}(\mathcal{H})$.

To compute the dimension of the coadjoint orbit in the dual space to $\mathfrak{k}=\mathfrak{s u}{ }_{N} \oplus \mathfrak{s u}{ }_{N}$ associated with $\left|\Psi^{\prime}\right\rangle$ via the moment map $\mu$ let us calculate

$$
\begin{gathered}
\mu_{(A, B)}\left(\sum_{i=1}^{N} p_{i} e_{i} \otimes e_{i}\right)=\left\langle\sum_{i=1}^{N} p_{i} e_{i} \otimes e_{i} \mid(A \otimes I+I \otimes B)\left(\sum_{j=1}^{N} p_{j} e_{j} \otimes e_{j}\right)\right\rangle \\
=\sum_{i, j=1}^{N}\left\langle p_{i} e_{i} \otimes e_{i} \mid p_{i} A e_{j} \otimes e_{j}+p_{j} e_{j} \otimes B e_{j}\right\rangle=\sum_{i=1}^{N} p_{i}^{2}\left(\left\langle e_{i} \mid A e_{i}\right\rangle+\left\langle e_{i} \mid B e_{i}\right\rangle\right)
\end{gathered}
$$

with $(A, B) \in \mathfrak{s u}_{N} \oplus \mathfrak{s u}_{N}$. It is easy to see (using the standard basis of $\mathfrak{s u}_{N}$ ) that in fact the $S V D$ transfers our state $|\Psi\rangle$ into a state $\left|\Psi^{\prime}\right\rangle$ such that $\mu\left(\left|\Psi^{\prime}\right\rangle\right) \in \mathfrak{t}^{*}$, where $\mathfrak{t}^{*}$ is the dual space to the Cartan subalgebra $\mathfrak{t}$ of $\mathfrak{k}$. Of course every coadjoint orbit is passing through at least one point from $t^{*}$ (usually, a coadjoint orbit contains more than one point from $t^{*}$, all these points lie on the orbit of the Weyl group). This fact could be seen as a geometrical interpretation of the SVD, but in contrast to the SDV itself, it remains true in multipartite cases. We will use it in the following sections. Going back to our considerations we know that $\mu\left(\left|\Psi^{\prime}\right\rangle\right)$ is determined by the action on $t$ which is generated by $I \otimes H, H \otimes I$, where $H$ belongs to the Cartan subalgebra of $\mathfrak{s u}_{N}$. Using an invariant scalar product on $\mathfrak{k}$ given by $\operatorname{Tr} A B$, we can find an element $(X, Y) \in \mathfrak{t}$ such that $\mu\left(\left|\Psi^{\prime}\right\rangle\right)=\alpha_{(X, Y)}$. To compute this we use the standard basis for $\mathfrak{t}$ given by $H_{1}=\operatorname{diag}(1,-1,0, \ldots, 0), \ldots, H_{N-1}=\operatorname{diag}(0,0, \ldots, 1,-1)$. We have

$$
\begin{aligned}
& \mu_{\left(H_{k}, 0\right)}\left(\left|\Psi^{\prime}\right\rangle\right)=\operatorname{tr}\left((X \otimes I+I \otimes Y) H_{k} \otimes I\right)=\operatorname{tr}\left(X H_{k}\right), \\
& \mu_{\left(0, H_{k}\right)}\left(\left|\Psi^{\prime}\right\rangle\right)=\operatorname{tr}\left((X \otimes I+I \otimes Y) I \otimes H_{k}\right)=\operatorname{tr}\left(Y H_{k}\right),
\end{aligned}
$$

but we also know that

$$
\mu_{\left(H_{k}, 0\right)}\left(\left|\Psi^{\prime}\right\rangle\right)=\sum_{i=1}^{N} p_{i}^{2}\left(e_{i} \mid H_{k} e_{i}\right)=p_{k}^{2}-p_{k+1}^{2}=\mu_{\left(0, H_{k}\right)}\left(\left|\Psi^{\prime}\right\rangle\right) .
$$

It is easy to see now that $X=Y$ and

$$
X=Y=\operatorname{diag}\left(-\frac{1}{N}+p_{1}^{2},-\frac{1}{N}+p_{2}^{2}, \ldots,-\frac{1}{N}+p_{N}^{2}\right)
$$


To compute the dimension of the coadjoint orbit through $\mu\left(\left|\Psi^{\prime}\right\rangle\right)$ notice that if $U_{1} \otimes U_{2} \in$ $G$ then

$$
U_{1} \otimes U_{2}(X \otimes I+I \otimes X) U_{1}^{\dagger} \otimes U_{2}^{\dagger}=U_{1} X U_{1}^{\dagger} \otimes I+I \otimes U_{2} X U_{2}^{\dagger}
$$

Hence, to obtain the dimension of the coadjoint orbit we need to compute the dimension of the stabilizer subgroup of $X$ by the adjoint action. It is easy to see that $X$ is stabilized by any matrix of the form:

$$
U=\left(\begin{array}{llll}
u_{0} & & & \\
& u_{1} & & \\
& & \ddots & \\
& & & u_{K}
\end{array}\right),
$$

where $u_{0}, u_{1}, \ldots, u_{K}$ are arbitrary unitary operators from $U\left(m_{0}\right), U\left(m_{1}\right), \ldots, U\left(m_{K}\right)$ and the value of $\operatorname{det}\left(u_{0}\right)$ is fixed by demanding that $U$ is special unitary. The dimension of the coadjoint orbit through $\mu\left(\left|\Psi^{\prime}\right\rangle\right)$ is thus

$$
\operatorname{dim}(\mu(\mathcal{O}))=\left(2 N^{2}-2\right)-\left(2 \sum_{n=0}^{K} m_{n}^{2}-2\right)=2 N^{2}-2 \sum_{n=0}^{K} m_{n}^{2}
$$

Now we are able to compute the dimension $D(|\Psi\rangle)$ of the degeneracy subspaces (fibers of the moment map),

$$
D(|\Psi\rangle)=\operatorname{dim}(\mathcal{O})-\operatorname{dim}(\mu(\mathcal{O}))=\sum_{n=1}^{K} m_{n}^{2}-1
$$

and we see that the orbit through $|\Psi\rangle$ is symplectic if and only if in the $S V D$ decomposition we get a diagonal matrix with only one non zero entry.

Fact 5. In the case of two identical but distinguishable particles there is only one symplectic orbit in the projective space $\mathbb{P}\left(\mathbb{C}^{N} \otimes \mathbb{C}^{N}\right)$. This orbit contains all separable states and is Kähler. Orbits through entangled states are not symplectic.

Knowing this and making use of Corollary 1 we arrive at

Fact 6. In the case of two identical but distinguishable particles the dimension of the degeneracy space $D(|\Psi\rangle)=\sum_{n=1}^{K} m_{n}^{2}-1$ gives a well defined entanglement measure.

\section{Three Particle Case}

As already mentioned the SVD has no generalization to multiple tensor products corresponding to multiparticle cases. Nevertheless we may apply some methods from the previous section if we look at the SVD from a slightly different point of view. Let us namely ask the question about necessary conditions for a state $|\Psi\rangle$ (66) to be sent by 
the moment map $\mu$ to an element of $\mathfrak{t}^{*}$ represented by $X \otimes I+I \otimes Y \in \mathfrak{t}$ upon the identification of $\mathfrak{t}^{*}$ and $\mathfrak{t}$ through the invariant scalar product on $\mathfrak{k}$. We have

$$
\begin{aligned}
\mu_{(A, B)}(|\Psi\rangle) & =\left\langle\sum_{i, j=1}^{N} C_{i j} e_{i} \otimes e_{j} \mid(A \otimes I+I \otimes B) \sum_{m, n=1}^{N} C_{m n} e_{m} \otimes e_{n}\right\rangle \\
& =\sum_{m, n=1}^{N} \sum_{i, j=1}^{N} \bar{C}_{i j} C_{m n}\left(\delta_{n j}\left\langle e_{i} \mid A e_{m}\right\rangle+\delta_{i m}\left\langle e_{j} \mid B e_{n}\right\rangle\right) \\
& =\sum_{i, j, m=1}^{N} \bar{C}_{i j} C_{m j}\left\langle e_{i} \mid A e_{m}\right\rangle+\sum_{i, j, n=1}^{N} \bar{C}_{i j} C_{i n}\left\langle e_{j} \mid B e_{n}\right\rangle \\
& =\sum_{i, m=1}^{N}\left(C C^{\dagger}\right)_{m i}\left\langle e_{i} \mid A e_{m}\right\rangle+\sum_{j, n=1}^{N}\left(C^{\dagger} C\right)_{j n}\left\langle e_{j} \mid B e_{n}\right\rangle \\
& =\sum_{i, j=1}^{N}\left(C C^{\dagger}\right)_{j i}\left\langle e_{i} \mid A e_{j}\right\rangle+\left(C^{\dagger} C\right)_{j i}\left\langle e_{j} \mid B e_{i}\right\rangle .
\end{aligned}
$$

In the following we will denote by $E_{i j}$ the matrix with zero entries everywhere except 1 on the $(i, j)$ position. Matrices $i\left(E_{i j}+E_{j i}\right)$ and $E_{i j}-E_{j i}$ supplemented by the previously defined standard basis elements of $\mathfrak{t}$ constitute a standard basis of $\mathfrak{k}$. Taking now $A$ and $B$ of the form $i\left(E_{i j}+E_{j i}\right)$ and $E_{i j}-E_{j i}$ which do not belong to $t$ but are from $\mathfrak{k}$, we must have

$$
\begin{aligned}
& \sum_{i, j=1}^{N}\left(C C^{\dagger}\right)_{j i}\left(e_{i} \mid\left(E_{k l}+E_{l k}\right) e_{j}\right)=0, \\
& \sum_{i, j=1}^{N}\left(C C^{\dagger}\right)_{j i}\left(e_{i} \mid\left(E_{k l}-E_{l k}\right) e_{j}\right)=0,
\end{aligned}
$$

and the same for $C^{\dagger} C$. Notice that

$$
\left\langle e_{i} \mid\left(E_{k l} \pm E_{l k}\right) e_{j}\right\rangle=\delta_{l j}\left(e_{i} \mid e_{k}\right) \pm \delta_{k j}\left(e_{i} \mid e_{l}\right)=\delta_{l j} \delta_{i k} \pm \delta_{k j} \delta_{i l},
$$

hence,

$$
\begin{aligned}
\left(C C^{\dagger}\right)_{l k}+\left(C C^{\dagger}\right)_{k l} & =0, \\
\left(C C^{\dagger}\right)_{l k}-\left(C C^{\dagger}\right)_{k l} & =0,
\end{aligned}
$$

and the same equations are fulfilled by $C^{\dagger} C$. It means that both $C C^{\dagger}$ and $C^{\dagger} C$ are diagonal. From linear algebra we know that the spectra of $\left(C C^{\dagger}\right)$ and $\left(C^{\dagger} C\right)$ are the same. Using this property and an additional freedom of a unitary action which permutes elements on the diagonal we notice that it is always possible to have $C C^{\dagger}=C^{\dagger} C$ (i.e., $C$ is a normal operator) and thus $X=Y$ in $X \otimes I+I \otimes Y$ for the corresponding image of the moment map. Let thus

$$
C^{\dagger} C=\left(\begin{array}{cccc}
0 I_{m_{0}} & & & \\
& v_{1} I_{m_{1}} & & \\
& & \ddots & \\
& & & v_{K} I_{m_{k}}
\end{array}\right),
$$


where $I_{n}$ is the unit $n \times n$ matrix. Then

$$
C=\left(\begin{array}{cccc}
0 & & & \\
& \sqrt{v_{1}} u_{1} & & \\
& & \ddots & \\
& & & \sqrt{v_{K}} u_{K}
\end{array}\right)
$$

where $m_{1}, \ldots, m_{k}$ are dimensions of degeneracy of $v_{1}, \ldots, v_{K}$, respectively and $u_{n}$ are $m_{n} \times m_{n}$ unitary matrices. Among all matrices (81) there is one which is diagonal and corresponds to the SVD. In this way we proved the existence of the SVD for any state using the fact that each adjoint orbit intersects the Cartan subalgebra $t$. The second important observation is that all states (81) are sent by the moment map into the same point $X \otimes I+I \otimes X$, and therefore constitute the fiber of the moment map. The dimension of this fiber is $\sum_{n=1}^{K} m_{n}^{2}-1$, which is exactly $D(|\Psi\rangle)$ from the previous section.

To a certain point we may repeat the reasoning in a multipartite case. Thus, e.g. for a general three-particle state,

$$
|\Psi\rangle=\sum_{i, j, k=1}^{N} C_{i j k} e_{i} \otimes e_{j} \otimes e_{k}
$$

the action of the moment map on $|\Psi\rangle$ gives:

$$
\begin{aligned}
& \mu_{(A, B, D)}(|\Psi\rangle) \\
& =\left\langle\sum_{i, j, k=1}^{N} C_{i j k} e_{i} \otimes e_{j} \otimes e_{k} \mid(A \otimes I \otimes I+I \otimes B \otimes I+I \otimes I \otimes D) \sum_{m, n, l=1}^{N} C_{m n l} e_{m} \otimes e_{n} \otimes e_{l}\right\rangle \\
& =\sum_{m, n, l=1}^{N} \sum_{i, j, k=1}^{N} \bar{C}_{i j k} C_{m n l}\left\langle e_{i} \otimes e_{j} \otimes e_{k} \mid A e_{m} \otimes e_{n} \otimes e_{l}+e_{m} \otimes B e_{n} \otimes e_{l}+e_{m} \otimes e_{n} \otimes D e_{l}\right\rangle \\
& =\sum_{m, n, l=1}^{N} \sum_{i, j, k=1}^{N} \bar{C}_{i j k} C_{m n l}\left(\delta_{j n} \delta_{k l}\left\langle e_{i} \mid A e_{m}\right\rangle+\delta_{i m} \delta_{k l}\left\langle e_{j} \mid B e_{n}\right\rangle+\delta_{i m} \delta_{j n}\left\langle e_{k} \mid D e_{l}\right\rangle\right) \\
& \left.=\sum_{i, j, k, m=1}^{N} \bar{C}_{i j k} C_{m j k}\left\langle e_{i} \mid A e_{m}\right\rangle+\bar{C}_{i j k} C_{i m k}\left\langle e_{j} \mid B e_{m}\right\rangle+\bar{C}_{i j k} C_{i j m}\left\langle e_{k} \mid D e_{m}\right\rangle\right) .
\end{aligned}
$$

Again, we want to find conditions for $C_{i j k}$ under which $\mu_{(A, B, D)}(|\Psi\rangle)$ belongs to $\mathfrak{t}^{*}$. Substituting for $A, B$, and $D$ basis elements from $\mathfrak{k}-\mathfrak{t}$ and again using (80) we get:

$$
\begin{aligned}
& \sum_{j, k=1}^{N} \bar{C}_{n j k} C_{l j k}+\sum_{j, k=1}^{N} \bar{C}_{l j k} C_{n j k}=0, \\
& \sum_{j, k=1}^{N} \bar{C}_{n j k} C_{l j k}-\sum_{j, k=1}^{N} \bar{C}_{l j k} C_{n j k}=0,
\end{aligned}
$$


and similarly two pairs of equations for the other combination of indices. If we now define

$$
\begin{aligned}
& \left(C^{1}\right)_{n l}=\sum_{j, k=1}^{N} \bar{C}_{n j k} C_{l j k}, \\
& \left(C^{2}\right)_{n l}=\sum_{j, k=1}^{N} \bar{C}_{j n k} C_{j l k}, \\
& \left(C^{3}\right)_{n l}=\sum_{j, k=1}^{N} \bar{C}_{j k n} C_{j k l},
\end{aligned}
$$

the obtained conditions mean that the matrices $C^{1}, C^{2}, C^{3}$ are diagonal. In this case it is not generally true that $C^{1}=C^{2}=C^{3}$, so the corresponding state in $\mathrm{t}$ is $X \otimes I \otimes I+$ $I \otimes Y \otimes I+I \otimes I \otimes Z$ where, in general, $X \neq Y \neq Z$.

Up to now we know that any state $|\tilde{\Psi}\rangle=\sum_{i, j, k=1}^{N} \tilde{C}_{i j k} e_{i} \otimes e_{j} \otimes e_{k}$ can be taken by local unitary transformation $U_{1} \otimes U_{2} \otimes U_{3}$ to the state $|\Psi\rangle=\sum_{i, j, k=1}^{N} C_{i j k} e_{i} \otimes e_{j} \otimes e_{k}$, where the coefficients $C_{i j k}$ fulfill (83). This statement has a deeper physical meaning. The diagonal elements of $C^{1}, C^{2}, C^{3}$ constitute probabilities to obtain basis vectors $\left\{e_{i}\right\}$ in some local measurements performed on the state $|\Psi\rangle$. The conditions (83) say that any state can be transformed by local unitary transformation to the state which is determined by these local measurements. It is natural to ask now how to find such a unitary local transformation.

Let us consider the arbitrary state $|\tilde{\Psi}\rangle$. The action of $U \otimes V \otimes W$ gives:

$$
\begin{aligned}
U \otimes V \otimes W|\tilde{\Psi}\rangle & =\sum_{i, j, k=1}^{N} \tilde{C}_{i j k} U e_{i} \otimes V e_{j} \otimes W e_{k} \\
& =\sum_{i, j, k=1}^{N} \tilde{C}_{i j k} U_{\alpha i} V_{\beta j} W_{\gamma k} e_{\alpha} \otimes e_{\beta} \otimes e_{\gamma} .
\end{aligned}
$$

The matrices $\tilde{C}^{1}, \tilde{C}^{2}, \tilde{C}^{3}$ are generally not diagonal but by definition they are positive, hence Hermitian. This means there are unitary operators $U, V, W$ such that $U^{\dagger} \tilde{C}^{1} U, V^{\dagger} \tilde{C}^{2} V, W^{\dagger} \tilde{C}^{3} W$ are diagonal. If we take now

$$
C_{i j k}=\sum_{n, l, m=1}^{N} \tilde{C}_{n l m} U_{i n}^{T} V_{j l}^{T} W_{k m}^{T},
$$

then:

$$
\begin{aligned}
\left(C^{1}\right)_{n l} & =\sum_{j, k=1}^{N} \bar{C}_{n j k} C_{l j k}=\sum_{j, k=1}^{N} \overline{\tilde{C}}_{\alpha \beta \gamma} \bar{U}_{n \alpha}{ }_{n \alpha} \bar{V}_{j \beta}{ }_{j \beta} \bar{W}_{k \gamma} \tilde{C}_{a b c} U_{l a}^{T} V_{j b}^{T} W_{k c}^{T} \\
& =\sum_{j, k=1}^{N} \overline{\tilde{C}}_{\alpha \beta \gamma} \tilde{C}_{a b c} U_{n \alpha}^{\dagger} U_{a l} V_{b j} V_{j \beta}^{\dagger} W_{c k} W_{k \gamma}^{\dagger}=U_{n \alpha}^{\dagger} \overline{\tilde{C}}_{\alpha \beta \gamma} \tilde{C}_{a \beta \gamma} U_{a l} \\
& =U_{n \alpha}^{\dagger}\left(\tilde{C}^{1}\right)_{\alpha a} U_{a l}=\left(U^{\dagger} \tilde{C}^{1} U\right)_{n l},
\end{aligned}
$$


which is diagonal as we wanted. Similarly we show that $C^{2}$ and $C^{3}$ are diagonal as well. Now to compute the dimension of the fiber over $\mu(|\Psi\rangle)$ we need to find the dimension of the submanifold of states which are sent to $\mu(|\Psi\rangle)$. First we look at the coadjoint orbit through $\mu(|\Psi\rangle)$. As we know $\mu(|\Psi\rangle)$ can be represented by an element of $X \otimes I \otimes I+I \otimes Y \otimes I+I \otimes I \otimes Z \in \mathfrak{t}$. Using similar reasoning as in the case of two particles we obtain:

$$
\begin{aligned}
& X=\operatorname{diag}\left(-\frac{1}{N}+p_{11}^{2},-\frac{1}{N}+p_{12}^{2}, \ldots,-\frac{1}{N}+p_{1 N}^{2}\right), \\
& Y=\operatorname{diag}\left(-\frac{1}{N}+p_{21}^{2},-\frac{1}{N}+p_{22}^{2}, \ldots,-\frac{1}{N}+p_{2 N}^{2}\right), \\
& Z=\operatorname{diag}\left(-\frac{1}{N}+p_{31}^{2},-\frac{1}{N}+p_{32}^{2}, \ldots,-\frac{1}{N}+p_{3 N}^{2}\right),
\end{aligned}
$$

where $\left\{p_{11}^{2}, p_{12}^{2}, \ldots, p_{1 N}^{2}\right\},\left\{p_{21}^{2}, p_{22}^{2}, \ldots, p_{2 N}^{2}\right\}$ and $\left\{p_{31}^{2}, p_{32}^{2}, \ldots, p_{3 N}^{2}\right\}$ constitute the spectra of $C^{1}, C^{2}$ and $C^{3}$, respectively. The dimension of this orbit can be easily computed knowing that $\operatorname{Stab}(\mu(|\Psi\rangle))$ consists of matrices $U \otimes V \otimes W$ and

$$
\begin{aligned}
& U=\left(\begin{array}{cccc}
u_{1,0} & & & \\
& u_{1,1} & & \\
& & \ddots & \\
& & & u_{1, K_{1}}
\end{array}\right), \quad V=\left(\begin{array}{cccc}
v_{2,0} & & & \\
& u_{2,1} & & \\
& & \ddots & \\
& & & u_{2, K_{2}}
\end{array}\right) \text {, } \\
& W=\left(\begin{array}{cccc}
w_{3,0} & & & \\
& w_{3,1} & & \\
& & \ddots & \\
& & & w_{3, K_{3}}
\end{array}\right)
\end{aligned}
$$

where $K_{i}$ is the number of eigenspaces of $C^{i}$ corresponding to different eigenvalues, $m_{i, n}$ are their dimensions and $u_{i, n} \in U\left(m_{i, n}\right)$. The stabilizer of this orbit has the dimension:

$$
\operatorname{dim}(\operatorname{Stab}(\mu(|\Psi\rangle)))=\sum_{n=0}^{K_{1}} m_{1, n}^{2}+\sum_{n=0}^{K_{2}} m_{2, n}^{2}+\sum_{n=0}^{K_{3}} m_{3, n}^{2}-3
$$

Hence,

$$
\operatorname{dim}(\mu(\mathcal{O}))=\left(3 N^{2}-3\right)-\left(\sum_{n=0}^{K_{1}} m_{1, n}^{2}+\sum_{n=0}^{K_{2}} m_{2, n}^{2}+\sum_{n=0}^{K_{3}} m_{3, n}^{2}-3\right) .
$$

The dimension of fiber can be computed as:

$$
\operatorname{dim}(D(|\Psi\rangle))=\operatorname{dim}(\operatorname{Stab}(\mu(|\Psi\rangle)))-\operatorname{dim}(\operatorname{Stab}(|\Psi\rangle)) .
$$

Notice that if $C^{1}, C^{2}, C^{3}$ have nontrivial kernels then in the decomposition of $\Psi$ there are no elements $e_{i} \otimes e_{j} \otimes e_{k}$ where $e_{i} \in \operatorname{Ker}\left(C^{1}\right)$ or $e_{j} \in \operatorname{Ker}\left(C^{2}\right)$ or $e_{j} \in \operatorname{Ker}\left(C^{3}\right)$. This means that acting on $|\Psi\rangle$ by unitary operators from $\operatorname{Stab}(\mu(|\Psi\rangle))$ which can be restricted to the kernels of $C^{1}, C^{2}, C^{3}$ we do not change the state $|\Psi\rangle$. We find thus an upper bound for the dimension of the degeneracy space as

$$
\sum_{n=1}^{K_{1}} m_{1, n}^{2}+\sum_{n=1}^{K_{2}} m_{2, n}^{2}+\sum_{n=1}^{K_{3}} m_{3, n}^{2}-3
$$


The dimension of a fiber is at least

$$
\max \left\{\sum_{n=1}^{K_{1}} m_{1, n}^{2}, \sum_{n=1}^{K_{2}} m_{2, n}^{2}, \sum_{n=1}^{K_{3}} m_{3, n}^{2}\right\}-1 .
$$

Indeed, the conditions (83) allow us to write the state $|\Psi\rangle$ as

$$
|\Psi\rangle=\sum_{i}^{N} p_{1 i} e_{i} \otimes v_{i}
$$

where

$$
v_{i}=\sum_{j k} \frac{1}{p_{1 i}} C_{i j k} e_{j} \otimes e_{k}, \quad i=1, \ldots, N
$$

constitute a set of orthonormal vectors. We can treat (92) as a bipartite decomposition of $\Psi$ in the orthonormal bases $\left\{e_{i}\right\}$ and $\left\{v_{i}\right\}$. In these bases $\Psi$ is thus represented by the matrix $\check{C}$,

$$
\check{C}=\left(\begin{array}{llll}
0 I_{m_{10}} & & & \\
& \check{p}_{1} I_{m_{1,1}} & & \\
& & \ddots & \\
& & & \check{p}_{K_{1}} I_{m_{1, K_{1}}}
\end{array}\right),
$$

where $\check{p}_{i}$ are different eigenvalues $p_{1 i}$. Application of $U \otimes I \otimes I \in \operatorname{Stab}(\mu(|\Psi\rangle))$ yields:

$$
\check{C}^{\prime}=\left(\begin{array}{cccc}
0 u_{10} & & & \\
& \check{p}_{1} u_{11} & & \\
& & \ddots & \\
& & & \check{p}_{K_{1}} u_{1, K_{1}}
\end{array}\right) .
$$

Clearly, the matrices $\check{C}^{\prime}$ of the above form constitute a manifold of dimension $\sum_{n=1}^{K_{1}} m_{1, n}^{2}-1$. In the case of two particles this is the whole fiber because acting with $U \otimes I$ and $I \otimes V$ we get exactly the same manifold. For multipartite systems, like the three particle case we consider, we have to take into account that acting with $I \otimes V \otimes I$ and $I \otimes I \otimes W$ may produce manifolds of larger dimensionalities which leads thus to the estimate (91). Summing up we have

$$
\max \left\{\sum_{n=1}^{K_{1}} m_{1, n}^{2}, \sum_{n=1}^{K_{2}} m_{2, n}^{2}, \sum_{n=1}^{K_{3}} m_{3, n}^{2}\right\}-1 \leq D(|\Psi\rangle) \leq \sum_{n=1}^{K_{1}} m_{1, n}^{2}+\sum_{n=1}^{K_{2}} m_{2, n}^{2}+\sum_{n=1}^{K_{3}} m_{3, n}^{2}-3 .
$$

Thus an orbit is symplectic if and only if

$$
\sum_{n=1}^{K_{1}} m_{1, n}^{2}=1, \quad \sum_{n=1}^{K_{2}} m_{2, n}^{2}=1, \quad \sum_{n=1}^{K_{3}} m_{3, n}^{2}=1
$$


But this means that the state $|\Psi\rangle$ is separable because it reduces to one of the states $e_{i} \otimes e_{j} \otimes e_{k}$. If the state is separable then of course by local operations we can transform it to the state $e_{1} \otimes e_{1} \otimes e_{1}$ and then (96) is fulfilled. In this way we found an easy way to check if a state is separable and showed that it is equivalent to the fact that the associated orbit is symplectic. We also have an estimate for dimensions of degeneracy spaces for entangled states. A generalization to cases of more than three particles is straightforward. So we have following

Theorem 5. In the case of L identical but distinguishable particles there is only one symplectic orbit in the projective space $\mathbb{P}\left(\bigotimes_{n=1}^{L} \mathbb{C}^{N}\right)$. This orbit contains all separable states and is Kähler. Orbits through entangled states are not symplectic.

Knowing this and making use of Corollary 1 we arrive at

Fact 7. In the case of $L$ identical but distinguishable particles the dimension of the degeneracy space $D(|\Psi\rangle)$ gives a well defined entanglement measure. For any state $|\Psi\rangle$ the estimate for this measure is given by a formula analogous to (95).

\section{Indistinguishable Particles}

The Kostant-Sternberg theorem can be directly applied also to indistinguishable particles, i.e., bosons and fermions. For $L$ bosons the relevant group is $K=S U(N)$ represented in $V=\operatorname{Sym}^{L}\left(\mathbb{C}^{N}\right)$. As above we want to check which orbits of $K$-action are symplectic in the projective space $\mathbb{P}(V)$. The best way to understand the problem is to do some nontrivial example and then generalize the obtained result. To this end let us consider the simplest case of $L=2$ and $N=3$, i.e., the representation of $S U(3)$ in $\operatorname{Sym}^{2}\left(\mathbb{C}^{3}\right)$. First we notice that the representation of $S U(N)$ in $\operatorname{Sym}^{2}\left(\mathbb{C}^{N}\right)$ is irreducible [18]. From the Kostant-Sternberg theorem it follows that it is enough to investigate the structure of the $\mathfrak{s l}_{3}(\mathbb{C})$ representation on $\operatorname{Sym}^{2}\left(\mathbb{C}^{N}\right)$. The $\mathfrak{g}=\mathfrak{s l}_{3}(\mathbb{C})$ algebra is eight-dimensional and can be decomposed as $\mathfrak{g}=\mathfrak{n}_{-} \oplus \mathfrak{h} \oplus \mathfrak{n}_{+}$, where $\mathfrak{h}$ is the Cartan subalgebra consisting of traceless diagonal matrixes and $\mathfrak{n}_{+}=\operatorname{Span}\left(E_{12}, E_{13}, E_{23}\right), \mathfrak{n}_{-}=$ $\operatorname{Span}\left(E_{21}, E_{31}, E_{32}\right)$. We define three linear functionals $L_{i}: \mathfrak{h} \rightarrow \mathbb{C}$,

$$
L_{i}\left(\operatorname{diag}\left(a_{1}, a_{2}, a_{3}\right)\right)=a_{i}, \quad i \in\{1,2,3\} .
$$

Let us choose a basis $B$ in $\operatorname{Sym}^{2}\left(\mathbb{C}^{3}\right), B=\left\{e_{1} \otimes e_{1}, e_{2} \otimes e_{2}, e_{3} \otimes e_{3}, e_{1} \otimes e_{2}+\right.$ $\left.e_{2} \otimes e_{1}, e_{1} \otimes e_{3}+e_{3} \otimes e_{1}, e_{2} \otimes e_{3}+e_{3} \otimes e_{2}\right\}$ where $e_{i} \in \mathbb{C}^{3}$ are the standard basis vectors. The action of the Lie algebra $\mathfrak{g}$ on $\operatorname{Sym}^{2}\left(\mathbb{C}^{3}\right)$ is the usual action of the tensor product of representations. Construction of the representation of $\mathfrak{s l}_{3}(\mathbb{C})$ on $\operatorname{Sym}^{2}\left(\mathbb{C}^{3}\right)$ is straightforward, we take the vector $e_{1} \otimes e_{1}$ which is the highest weight vector (it is an eigenvector of all elements in $\mathfrak{h}$ and it is annihilated by $\left.\mathfrak{n}_{+}\right)$, and we act on it with operators from $\mathfrak{n}_{-}$. As a result we obtain a decomposition of $V=\operatorname{Sym}^{2}\left(\mathbb{C}^{3}\right)$ into the direct sum $V=\bigoplus V_{\lambda}$, where the one-dimensional weight spaces $V_{\lambda}$ are spanned by the basis vectors of $B$. The weights $\lambda \in \mathfrak{h}^{*}$ can be now calculated as ${ }^{1}$

$$
\begin{gathered}
H\left(e_{i} \otimes e_{i}\right)=2 L_{i}(H) e_{i} \otimes e_{i}, \quad i=1,2,3, \\
H\left(e_{i} \otimes e_{j}+e_{j} \otimes e_{i}\right)=\left(L_{i}+L_{j}\right)(H)\left(e_{i} \otimes e_{j}+e_{j} \otimes e_{i}\right) .
\end{gathered}
$$

\footnotetext{
1 Remember that we represent $\mathfrak{k}$ in the symmetric tensor product, hence $H\left(e_{i} \otimes e_{i}\right)$ has the meaning of $(H \otimes I+I \otimes H)\left(e_{i} \otimes e_{i}\right)=H e_{i} \otimes e_{i}+e_{i} \otimes H e_{i}$, etc.
} 
We know that only orbits passing through weight vectors might be symplectic. We have the following $\mathfrak{s l}_{2}(\mathbb{C})$ triples in $\mathfrak{s l}_{3}(\mathbb{C}):\left(E_{i j}, E_{j i}, H_{i j}=\left[E_{i j}, E_{j i}\right]\right)$. The orbit through a weight vector $v$ with a weight $\lambda$ is symplectic if and only if for every operator from $\mathfrak{n}_{+}$ the following implication is true: $\lambda\left(H_{i j}\right)=0 \Rightarrow E_{i j}(v)=0=E_{j i}(v)$. There are two cases to consider,

- Vectors of the form $e_{i} \otimes e_{i}, i=1,2,3$. The weight of the $e_{i} \otimes e_{i}$ state is $2 L_{i}$ so $2 L_{i}\left(H_{k j}\right)=0$ only when $k \neq i$ and $j \neq i$. In this case $E_{k j}\left(e_{i} \otimes e_{i}\right)=0$ because to give the nonzero result the matrix $E_{k j}$ must have one in the $i^{\text {th }}$ column. The corresponding orbit is thus symplectic. Obviously, all these vectors lie on the orbit through the highest weight vector $e_{1} \otimes e_{1}$.

- Vectors of the form $e_{i} \otimes e_{j}+e_{j} \otimes e_{i}$ where $i \neq j$. The weight of this vector is $L_{i}+L_{j}$ so $\left(L_{i}+L_{j}\right)\left(H_{k l}\right)=0$ only if $k=i$ and $j=l$. In this case $E_{i j}\left(e_{i} \otimes e_{j}+e_{j} \otimes e_{i}\right) \neq 0$ because $E_{i j} e_{j} \neq 0$. In conclusion the orbit through $e_{i} \otimes e_{j}+e_{j} \otimes e_{i}$ is not symplectic.

Let us now return to the problem mentioned in Sect. 2.2. If we define nonentangled bosonic states as antisymmetrizations of simple tensors (or, more precisely, as corresponding points in the projective space) then we clearly have two, inequivalent from the geometric point of view, types of nonentanglement. Nonentangled states of two different types are not connected by local unitary transformations which is in contrast to the familiar situation of distinguishable particles and intuitions built upon the fact that all separable states of distinguishable particles can be obtained from a single one by local transformations. Although this is obviously acceptable, it remains an open problem: what is a physical meaning of two different types of nonentanglement. If, instead, we adopt the second definition identifying nonentangled bosonic states as points in the projective space corresponding to tensor products of the same vector, we encounter the same situation as in the case of distinguishable particles - the nonentangled states form a unique symplectic orbit, and the degeneracy of the symplectic form can be used as a measure of entanglement for entangled states.

The case of fermions does not lead to any ambiguities of the above type. Calculations similar to those made for bosons lead to a conclusion that nonentangled states form the unique symplectic orbit. Indeed, let us consider as an example $K=S U(N)$ and $V=\bigwedge^{2} \mathbb{C}^{N}$ corresponding to two fermions of spin $(N-1) / 2$ with the single-particle space $\mathcal{H}_{1}=\mathbb{C}^{N}$. In terms of the previously introduced standard bases $e_{i}$ and $E_{i j}$ adapted to $N$ dimensions, $V$ is spanned by $e_{k l}=e_{k} \otimes e_{l}-e_{l} \otimes e_{k}$, with $k<l$ the highest weight vector is $e_{12}$ and

$$
E_{i j} e_{k l}=\delta_{j l} e_{k i}+\delta_{j k} e_{i l},
$$

where we denote $e_{k l}=-e_{l k}$ for $k>l$. Acting by $E_{i j}$ with $i>j$ on $e_{12}$ we obtain the remaining weight vectors, which according to (98) are all of the form $e_{k l}$ (in fact with $l=1$ or 2), with weights $L_{i}+L_{j}$ (we extended in an obvious way the definition (96) to $N$ dimensions). As remarked $\left(L_{i}+L_{j}\right)\left(H_{k l}\right)=0$ implies $k=i, j=l$, but then $E_{i j} e_{k l}=0=E_{j i} e_{k l}$.

\section{Summary and Outlook}

We presented a geometric description of the set of pure states of composite quantum systems in terms of the natural symplectic structure in the space of states. Nonentangled states form a unique symplectic orbit through the highest weight vector of the appropriate representation of the group of local transformations whereas entangled states are 
characterized by the degeneracy of the symplectic form. The degeneracy can be thus used as a kind of geometric measure of entanglement. We were able to calculate the degeneracy in many relevant cases and give some estimates for the most general system of an arbitrary number of constituents with an arbitrary dimension of the single particle space. Let us remark that there exists a useful characterization of the highest weight vector orbits which allows to generalize and estimate effectively some other entanglement measures [19].

An obvious question is whether a method can be adapted to the case of mixed states. This problem, as well as applications of the obtained results to identifying, so called, locally unitary equivalent multiparticle states [20] and finding "canonical" forms of them we postpone to forthcoming publications.

Acknowledgements. The support by SFB/TR12 Symmetries and Universality in Mesoscopic Systems program of the Deutsche Forschungsgemeischaft and Polish MNiSW grant DFG-SFB/38/2007 is gratefully acknowledged.

Open Access This article is distributed under the terms of the Creative Commons Attribution Noncommercial License which permits any noncommercial use, distribution, and reproduction in any medium, provided the original author(s) and source are credited.

\section{Appendix}

A.1 Symplectic structure on coadjoint orbits. Let $K$ be a semisimple compact Lie group, $\mathfrak{k}$ its Lie algebra, and $\mathfrak{k}^{*}$ the dual space to $\mathfrak{k}$. The coadjoint action of $K$ on $\mathfrak{k}^{*}$ is given by

$$
\begin{gathered}
\operatorname{Ad}_{g}^{*}: \mathfrak{k}^{*} \rightarrow \mathfrak{k}^{*}, \\
\left\langle\operatorname{Ad}_{g}^{*} \alpha, Y\right\rangle=\left\langle\alpha, \operatorname{Ad}_{g^{-1}} Y\right\rangle=\left\langle\alpha, g^{-1} Y g\right\rangle, \quad g \in K, \quad Y \in \mathfrak{k}, \quad \alpha \in \mathfrak{k}^{*},
\end{gathered}
$$

where Ad is the adjoint action of $K$. It can be easily checked that (99) is well defined. The coadjoint orbit $\Omega_{\alpha}$ passing through $\alpha \in \mathfrak{k}^{*}$ is the orbit of the coadjoint action of $K$ on $\alpha$,

$$
\Omega_{\alpha}=\left\{\operatorname{Ad}_{g}^{*} \alpha: g \in K\right\} .
$$

Our goal now is to define a $K$-invariant symplectic form $\omega$ on $\Omega_{\alpha}$. Such a form acts on tangent vectors so it is reasonable to look first at their structure. Since $\mathfrak{k}^{*}$ is a vector space its tangent space at any point is again $\mathfrak{k}^{*}$. For any $X \in \mathfrak{k}$ let $\tilde{X} \in T_{\alpha} \Omega_{\alpha}$ be a vector tangent to the curve $t \mapsto \operatorname{Ad}_{\exp (t X)}^{*} \alpha$. We have then

$$
\langle\tilde{X}, Y\rangle=\left\langle\left.\frac{d}{d t}\right|_{t=0} \operatorname{Ad}_{\exp (t X)}^{*} \alpha, Y\right\rangle=\left.\frac{d}{d t}\right|_{t=0}\left\langle\alpha, \operatorname{Ad}_{\exp (-t X)} Y\right\rangle=\langle\alpha,[Y, X]\rangle,
$$

where $Y \in \mathfrak{k}$. Thus $\tilde{X}$ is an element of $\mathfrak{k}^{*}$ given by

$$
\tilde{X}=\langle\alpha,[\cdot, X]\rangle .
$$

It is now interesting to ask how this tangent vector transforms when pushed by an element $g \in K$, i.e. to consider the vector $g \tilde{X} \in T_{\mathrm{Ad}_{g}^{*} \alpha} \Omega_{\alpha}$ tangent to the curve $t \mapsto$ 
$\operatorname{Ad}_{g}^{*} \operatorname{Ad}_{\exp (t X)}^{*} \alpha$. We have

$$
\begin{aligned}
\langle g \tilde{X}, Y\rangle & =\left\langle\left.\frac{d}{d t}\right|_{t=0} \operatorname{Ad}_{g}^{*} \operatorname{Ad}_{\exp (t X)}^{*} \alpha, Y\right\rangle=\left.\frac{d}{d t}\right|_{t=0}\left\langle\alpha, \operatorname{Ad}_{\exp (-t X)} \operatorname{Ad}_{g^{-1}} Y\right\rangle \\
& =\left\langle\alpha,\left[\operatorname{Ad}_{g^{-1}} Y, X\right]\right\rangle=\left\langle\alpha,\left[\operatorname{Ad}_{g^{-1}} Y, \operatorname{Ad}_{g^{-1}} \operatorname{Ad}_{g} X\right]\right. \\
& =\left\langle\alpha, \operatorname{Ad}_{g^{-1}}\left[Y, \operatorname{Ad}_{g} X\right]\right\rangle=\left\langle\operatorname{Ad}_{g}^{*} \alpha,\left[Y, \operatorname{Ad}_{g} X\right]\right\rangle .
\end{aligned}
$$

Hence $g \tilde{X}$ is an element of $\mathfrak{k}^{*}$ given by

$$
g \tilde{X}=\left\langle\operatorname{Ad}_{g}^{*} \alpha,\left[\cdot, \operatorname{Ad}_{g} X\right]\right\rangle .
$$

We may now define our symplectic form at any $\beta \in \Omega_{\alpha}$ as

$$
\omega_{\beta}(\tilde{X}, \tilde{Y})=\langle\beta,[X, Y]\rangle .
$$

The form is nondegenerate on $\Omega_{\alpha}$ because

$$
\forall Y \in \mathfrak{k} \quad\langle\beta,[X, Y]\rangle=0 \Leftrightarrow \forall Y \in \mathfrak{k} \quad\langle\tilde{X}, Y\rangle=0 \Leftrightarrow \tilde{X}=0 .
$$

It is also $K$-invariant because

$$
\begin{aligned}
\omega_{\operatorname{Ad}_{g}^{*} \beta}(g \tilde{X}, g \tilde{Y}) & =\left\langle\operatorname{Ad}_{g}^{*} \beta,\left[\operatorname{Ad}_{g} X, A d_{g} Y\right]\right\rangle=\left\langle\beta, A d_{g^{-1}} A d_{g}[X, Y]\right\rangle \\
& =\langle\beta,[X, Y]\rangle=\omega_{\beta}(\tilde{X}, \tilde{Y}) .
\end{aligned}
$$

Thus we need only to check if $\omega$ is closed. But we know that for any differential 2-form the following is true:

$$
\begin{aligned}
d \omega(\tilde{X}, \tilde{Y}, \tilde{Z})= & \tilde{X} \omega(\tilde{Y}, \tilde{Z})+\tilde{Y} \omega(\tilde{Z}, \tilde{X})+\tilde{Z} \omega(\tilde{X}, \tilde{Y}) \\
& -(\omega([\tilde{X}, \tilde{Y}], \tilde{Z})+\omega([\tilde{Y}, \tilde{Z}], \tilde{X})+\omega([\tilde{Z}, \tilde{X}], \tilde{Y}))
\end{aligned}
$$

Taking $\tilde{X}=\left\langle\operatorname{Ad}_{g}^{*} \beta,[\cdot, \operatorname{Ad}(g) X]\right\rangle$ and similarly for $\tilde{Y}$ and $\tilde{Z}$ we see that the first three terms in (108) vanish because $\omega$ is $K$-invariant. The sum of the next three terms is also zero due to the Jacobi identity. This way we arrive at a well defined symplectic form on $\Omega_{\alpha}$.

A.2 Kähler structure. Let us start with the definition of Kähler manifold.

Definition 2. Let $M$ be a complex manifold $\operatorname{dim}_{\mathbb{C}} M=n$ and let $\omega$ be a symplectic form on $M$ treated as a $2 n$-dimensional real manifold. Then $M$ is called a Kähler manifold if at every $p \in M$ the complex structure $i$ on $T_{p} M$ (multiplication by an imaginary unit) $\omega$ fulfills:

$$
\omega(i v, i w)=\omega(v, w)
$$

that is $i \in \operatorname{Sp}\left(T_{p} M\right)$ (the symplectic group of $\left.T_{p} M\right)$. 
Assume $M$ is a Kähler manifold. Then we can define a symmetric nondegenerate form $b$ on $M$,

$$
b(v, w)=\omega(v, i w)
$$

Indeed we have

$$
b(v, w)=\omega(v, i w)=\omega\left(i v, i^{2} w\right)=-\omega(i v, w)=\omega(w, i v)=b(w, v) .
$$

The form $b$ is nondegenerate because

$$
\forall w \in T_{p} M \quad b(v, w)=0 \Leftrightarrow \forall w \in T_{p} M \quad \omega(w, i v)=0 \Leftrightarrow v=0 .
$$

It is also $i$-invariant

$$
b(i v, i w)=\omega\left(i v, i^{2} w\right)=\omega(v, i w)=b(v, w) .
$$

We have now the following definition.

Definition 3. A Kähler structure on $M$ is called positive if and only if the corresponding symmetric form $b$ is positive.

It is straightforward to check that having such an $i$-invariant nondegenerate symmetric form $b$ on $M$ we can define the nondegenerate $i$-invariant antisymmetric 2-form by $\omega(v, w)=b(i v, w)$. We will need only one more theorem (see [12] for a detailed proof).

Theorem 6. Let $M$ be a positive Kähler manifold. Then any complex submanifold $S \subset$ $M$ is also a Kähler manifold.

The assumption that $M$ is a positive Kähler manifold (not just a Kähler manifold) is very important due to the fact that restriction of the symmetric form $b$ to $S$ is then nondegenerate and $i$-invariant. Now using $\left.b\right|_{\mathcal{O}}$ we can define a nondegenerate $i$-invariant antisymmetric 2 -form on $\mathcal{O}$ which is a restriction of $\omega$ defined on the whole $M$, and hence is symplectic.

A.2.1 Kähler structure on $\mathbb{P}(V)$. Consider a complex vector space $V, \operatorname{dim}_{\mathbb{C}} V=n$ with a Hermitian scalar product $(\cdot \mid \cdot)$. The complex projective space $\mathbb{P}(V)$ of $V$ is defined

$$
\mathbb{P}(V)=V / \sim
$$

where the equivalence $\sim$ is defined by

$$
v \sim w \Leftrightarrow w=\alpha v \quad \alpha \in \mathbb{C}^{*},
$$

where $\mathbb{C}^{*}=\mathbb{C} \backslash\{0\}$. The standard way to realize $\mathbb{P}(V)$ is by two steps:

$$
V \stackrel{a}{\longrightarrow} S(V) \stackrel{b}{\longrightarrow} \mathbb{P}(V),
$$

where step $a$ is a quotient by the dilation $v \sim \alpha v$ for $a \in \mathbb{R}^{*}$ and step $b$ is a quotient by the rotations $v \sim e^{i \phi} v$. The result of the quotient $a$ is the real sphere $S(V)=$ $\{v \in v:\|v\|=1\}$. The quotient $b$ gives $S(V) / S^{1}=S^{2 n-1} / S^{1}$, where $S^{1}$ represents the group of rotations. It is a well known fact that the complex projective space $\mathbb{P}(V)$ is a complex manifold and $\operatorname{dim}_{\mathbb{C}} \mathbb{P}(V)=n-1$. Let $\pi: V \backslash\{0\} \rightarrow \mathbb{P}(V)$ be the projection defined by equivalence $\sim$. The tangent space to $\mathbb{P}(V)$ at $z=\pi(v)$ 
is $T_{z} \mathbb{P}(V)=\pi_{v}\left(T_{v} V\right)$. It is a good question to ask what is $\pi_{v}(\xi)$, where $\xi \in T_{v} V$. Consider the curve $t \mapsto v(t) \in V, v(0)=v$. Then

$$
\xi=\left.\frac{d}{d t}\right|_{t=0} v(t)
$$

We first apply the map $a$ to $v(t)$. As a result we get a curve $t \mapsto \frac{v(t)}{\|v(t)\|} \in S(V)$. Applying the map $b$ amounts to getting rid of the rotation $e^{i \phi} v$, hence finally the curve in $\mathbb{P}(v)$ is given as

$$
t \mapsto \frac{v(t)}{\|v(t)\|}-v\left(\frac{v}{\|v\|} \mid \frac{v(t)}{\|v(t)\|}\right) .
$$

The tangent vector to this curve is thus

$$
\left.\frac{d}{d t}\right|_{t=0} \frac{v(t)}{\|v(t)\|}-\left.\frac{d}{d t}\right|_{t=0} v\left(\frac{v}{\|v\|} \mid \frac{v(t)}{\|v(t)\|}\right)=\frac{\xi}{\|v\|}-\frac{v}{\|v\|}\left(\frac{v}{\|v\|} \mid \frac{\xi}{\|v\|}\right) .
$$

Hence for any vector $\xi \in T_{v} V$, we obtain the corresponding vector $\pi_{v}(\xi) \in T_{\pi(v)} \mathbb{P}(V)$ as the orthogonal complement of $\frac{\xi}{\|v\|}$ to the subspace $\mathbb{C} v$ in the Hermitian scalar product $(\cdot \mid \cdot)$. Let us introduce a Hermitian scalar product on $\mathbb{P}(V)$ by

$$
h\left(\pi_{v}(\xi), \pi_{v}(\eta)\right)=\left(\frac{1}{\|v\|} \frac{\xi(v \mid v)-(v \mid \xi) v}{(v \mid v)} \mid \frac{1}{\|v\|} \frac{\eta(v \mid v)-(v \mid \eta) v}{(v \mid v)}\right),
$$

which, after some calculations, reduces to

$$
h\left(\pi_{v}(\xi), \pi_{v}(\eta)\right)=\frac{(\xi \mid \eta)(v \mid v)-(\xi \mid v)(v \mid \eta)}{(v \mid v)^{2}} .
$$

Of course, $h$ is a well defined, that is a nondegenerate, positive Hermitian form on $\mathbb{P} V$. Indeed, for any $z=\pi(v)$ we have $T_{z} \mathbb{P}(V)=(\mathbb{C} v)^{\perp}$. Knowing this we can introduce a nondegenerate antisymmetric 2 -form $\omega$ on $\mathbb{P}(V)$ as the imaginary part of $h(\cdot, \cdot)$,

$$
\omega\left(\pi_{v}(\xi), \pi_{v}(\eta)\right)=-\operatorname{Im} h\left(\pi_{v}(\xi), \pi_{v}(\eta)\right) .
$$

It is straightforward to check that $\omega$ is not only $i$-invariant but also $U(V)$-invariant. To check that it is also closed notice that $U(V)$ is acting transitively on $V$ which means the vectors $A v$, where $A \in u(V)$ span $T_{v} V$. Hence,

$$
\omega\left(\pi_{v}(A v), \pi_{v}(B v)\right)=-\operatorname{Im} \frac{(A v \mid B v)(v \mid v)-(A v \mid v)(v \mid B v)}{(v \mid v)^{2}}, \quad A, B \in u(V) .
$$

But $(A v \mid v)(v \mid B v)$ is real since $(A v \mid v)$ and $(v \mid B v)$ are imaginary (this is because $A^{\dagger}=$ $-A$ for all $A \in u(V))$. Hence,

$$
\omega\left(\pi_{v}(A v), \pi_{v}(B v)\right)=-\operatorname{Im} \frac{(A v \mid B v)}{(v \mid v)}=\frac{i([A, B] v \mid v)}{2(v \mid v)}, \quad A, B \in u(V) .
$$

Now making use of Eq. (108) for vector fields $\pi_{U v}\left(U A U^{*} U v\right)$ and similarly for $B$ and $C$ we find, by the same argument as for coadjoint orbits, that $d \omega=0$. This means $\mathbb{P}(V)$ is a Kähler manifold. It is also a positive Kähler manifold because the corresponding symmetric form $b$ given by

$$
b\left(\pi_{v}(\xi), \pi_{v}(\eta)\right)=-\operatorname{Re} h\left(\pi_{v}(\xi), \pi_{v}(\eta)\right),
$$

is clearly positive. 


\section{References}

1. Horodecki, R., Horodecki, P., Horodecki, M., Horodecki, K.: Quantum entanglement. Rev. Mod. Phys. 81, 865-942 (2009)

2. Werner, R.: Quantum states with Einstein-Podolsky-Rosen correlation admitting a hidden-variable model. Phys. Rev. A 40, 4277-4281 (1989)

3. Schliemann, J., Cirac, J.I., Kuś, M., Lewenstein, M., Loss, D.: Quantum correlations in two-fermion systems. Phys. Rev. A 64, 022303 (2001)

4. Eckert, K., Schliemann, J., Bruß, D., Lewenstein, M.: Quantum correlations in systems of identical particles. Ann. Phys. 299, 88-127 (2002)

5. Ghirardi, G., Marinatto, L., Weber, T.: Entanglement and properties of composite quantum systems, a conceptual and mathematical analysis. J. Stat. Phys. 108, 49-122 (2002)

6. Li, Y.S. Zeng, B., Liu, X.S., Long, G.L.: Entanglement in a two-identical-particle system. Phys. Rev. A 64, 054302 (2001)

7. Paškauskas, R., You, L.: Quantum correlations in two-boson wave functions. Phys. Rev. A 64, 042310 (2001)

8. Klyachko, A.: Dynamic symmetry approach to entanglement. http://arxiv.org/alos/0802.4008v1 [quant$\mathrm{ph}]$, (2008)

9. Bengtsson, I.: A curious geometrical fact about entanglement. AIP Conf. Proc. 962(1), 34-38 (2007)

10. Kuś, M., Bengtsson, I.: Classical quantum states. Phys. Rev. A 80, 022319 (2009)

11. Kostant, B., Sternberg, B.: Symplectic projective orbits. In: New directions in applied mathematics, papers presented April 25/26, 1980, on the occasion of the Case Centennial Celebration (Hilton, P. J., Young, G. S., eds.) New York: Springer, 1982, pp 81-84

12. Guillemin, V., Sternberg, S.: Symplectic techniques in physics. Cambridge: Cambridge University Press, (1984)

13. Perelomov, A.: Generalized coherent states and their applications. Springer: Heidelberg, 1986

14. Hall, B.C.: Lie groups, Lie algebras, and representations, an elementary introduction. New York: Springer, (2003)

15. Kirillov, A.A.: Lectures on the orbit method. Graduate Studies in Mathematics Vol. 64. Providence, RI: Amer. Math. Soc., 2004

16. Horn, R.A., Johnson, C.R.: Matrix analysis. Cambridge: Cambridge University Press, 1985

17. Sinołęcka, M.M., Życzkowski, K., Kuś, M.: Manifolds of equal entanglement for composite quantum system. Acta Phys. Pol. 33, 2081-2095 (2002)

18. Fulton, W., Harris, J.: Representation theory. A first course. New York: Springer, 1991

19. Kotowski, M., Kotowski, M., Kuś, M.: Universal nonlinear entanglement witnesses. Phys. Rev. A 81, 062318 (2010)

20. Kraus, B.: Local unitary equivalence of multipartite pure states. Phys. Rev. Lett. 104, 020504 (2010)

Communicated by M.B. Ruskai 